\begin{tabular}{|c|c|c|c|}
\hline Figure \# & $\begin{array}{l}\text { Figure title } \\
\text { One sentence only }\end{array}$ & $\begin{array}{l}\text { Filename } \\
\text { This should be the } \\
\text { name the file is saved as } \\
\text { when it is uploaded to } \\
\text { our system. Please } \\
\text { include the file } \\
\text { extension. i.e.: Smith_ED } \\
\text { Fig1.jpg }\end{array}$ & $\begin{array}{l}\text { Figure Legend } \\
\text { If you are citing a reference for the first time in these legends, } \\
\text { please include all new references in the Online Methods References } \\
\text { section, and carry on the numbering from the main References } \\
\text { section of the paper. }\end{array}$ \\
\hline Extended Data Fig. 1 & $\begin{array}{l}\text { Distribution of Form I' } \\
\text { Chloroflexi genomes. }\end{array}$ & Shih_EDFig1.eps & $\begin{array}{l}\text { Maximum likelihood phylogenetic tree of Chloroflexi using } \\
\text { ribosomal protein S3 (rpS3) as a marker gene. To map the } \\
\text { distribution of Form I' Rubisco genes onto genomes, all MAGs } \\
\text { were scanned for presence of both rpS3 and Form I' Rubisco. } \\
\text { MAGs containing Form I' Rubisco are highlighted in orange. } \\
\text { The scaffolds that encode RbcL vary in size substantially, } \\
\text { ranging up to } 106 \text { kbp in length (available as Supplementary } \\
\text { Data). At least partial genomic context could be determined in } \\
\text { most cases and the gene for phosphoribulokinase was } \\
\text { adjacent. In some cases, additional CBB Cycle pathway genes } \\
\text { were present in an operon with Rubisco, strongly supporting } \\
\text { the function of Rubisco in this pathway. In a subset of cases, } \\
\text { other pentose phosphate pathway genes were co-encoded. In } \\
\text { no case was there evidence for RbcS, either on the scaffold or } \\
\text { in the draft genome bin (where a bin was available). Gene } \\
\text { predictions were established via a standard annotation } \\
\text { pipeline }{ }^{50,51} \text { and augmented by HMM-based profiling and } \\
\text { domain analysis. }\end{array}$ \\
\hline Extended Data Fig. 2 & $\begin{array}{l}\text { In Form I-containing } \\
\text { Chloroflexi operons, } \\
r b c L \text { and } r b c S \text { are always } \\
\text { found next to each }\end{array}$ & Shih_EDFig2.eps & $\begin{array}{l}\text { Fragment operons from an example set of } 10 \text { Form I Rubisco- } \\
\text { containing Chloroflexi genomes shows that } r b c S \text { is always } \\
\text { found next to } r b c L \text {, similar to Form I Rubisco found in } \\
\text { Cyanobacteria and Proteobacteria }{ }^{11} \text {. Form I' Rubisco- }\end{array}$ \\
\hline
\end{tabular}




\begin{tabular}{|c|c|c|c|}
\hline & $\begin{array}{l}\text { other, unlike Form I'- } \\
\text { containing Chloroflexi } \\
\text { operons that lack rbcS. }\end{array}$ & & $\begin{array}{l}\text { containing Chloroflexi genomes do not contain small subunit } \\
\text { rbcS (Fig. 1b). Scaffold names are shown to the right of their } \\
\text { corresponding genome fragments. }\end{array}$ \\
\hline Extended Data Fig. 3 & PAGE analyses. & Shih_EDFig3.eps & 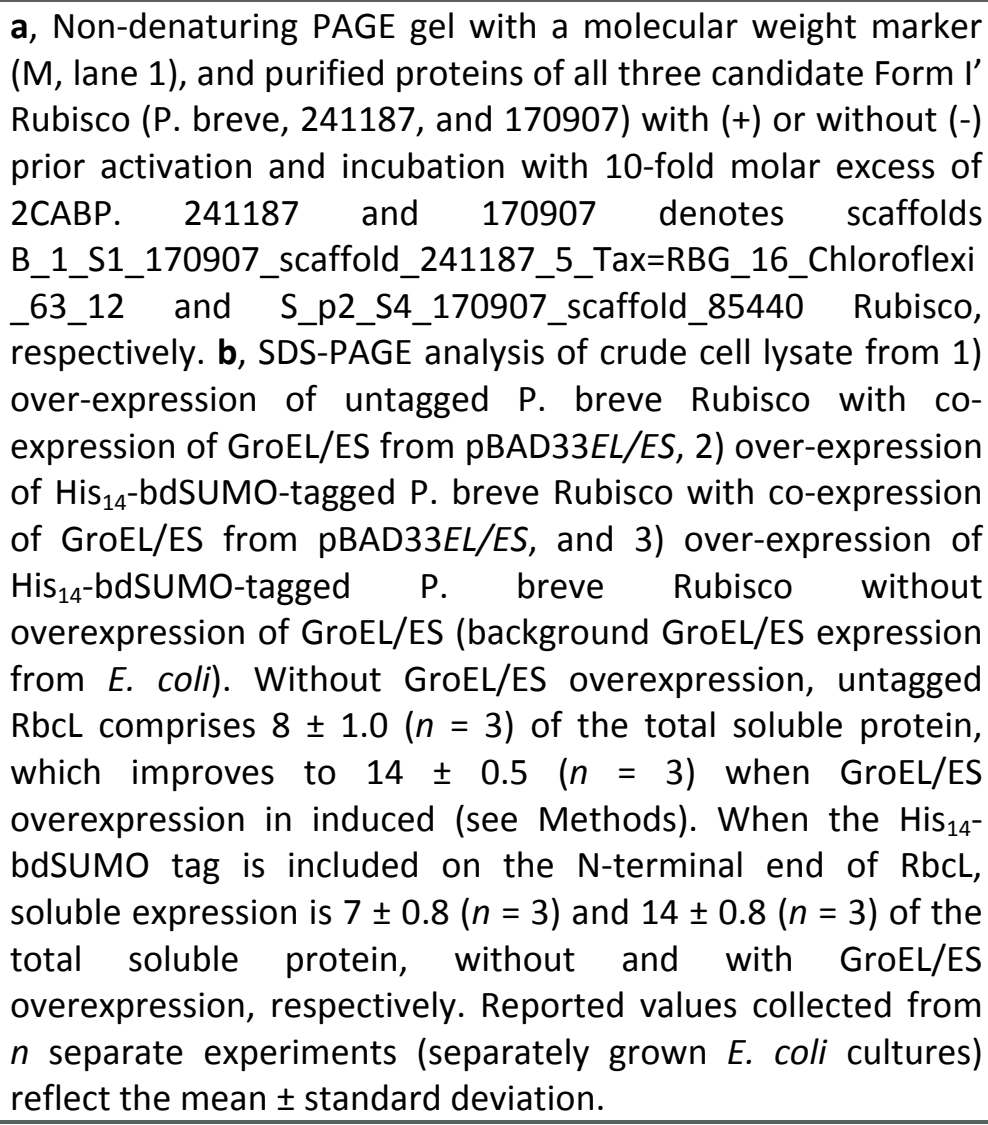 \\
\hline Extended Data Fig. 4 & $\begin{array}{l}\text { Form I Rubisco possess } \\
\text { a unique RbcL C- } \\
\text { terminal extension that } \\
\text { interacts with RbcS, } \\
\text { which is not found in } \\
\text { Form I' Rubisco. }\end{array}$ & Shih_EDFig4.tiff & $\begin{array}{l}\text { a, Sequence alignment of representative Rubisco RbcL } \\
\text { sequences from Forms I, I', II, II/III, IIIA and IIIb. Strictly } \\
\text { conserved residues have a red background, residues well } \\
\text { conserved within a group are indicated by red letters, and the } \\
\text { remaining residues are in black letters. Gaps are represented } \\
\text { by dots. Residue numbering along the top refers to P. breve } \\
\text { RbcL. Symbols above blocks of sequences correspond to the }\end{array}$ \\
\hline
\end{tabular}




\begin{tabular}{|c|c|c|c|}
\hline & & & $\begin{array}{l}\text { secondary structure of P. breve RbcL: } \alpha, \alpha \text {-helix; } \beta, \beta \text {-strand; } \eta \text {, } \\
\text { 310-helix. The secondary structure elements were named } \\
\text { according to Knight et al., 199052. The positions of loop } 6 \\
\text { (black dotted lined), the Form II/III-specific Rubisco assembly } \\
\text { domain (cyan line), and the Form I-specific C-terminal } \\
\text { extension (purple line) are indicated. The RbcX binding } \\
\text { domain-specific to Form IB Rubisco is boxed in pink. The } \\
\text { sequence alignment was created using the UniProt RbcL } \\
\text { sequences P22859 (Allochromatium vinosum), O85040 } \\
\text { (Halothiobacillus neapolitanus), A0A4D4IZ26 (Zea mays), } \\
\text { P00880 (Syn6301), Q1QH22 (Nitrobacter hamburgensis), } \\
\text { Q3IYC2 (Rhodobacter sphaeroides), P51226 (Porphyra } \\
\text { purpurea), Q9GGQ2 (Vaucheria litorea), E1IGS1 (Oscillochloris } \\
\text { trichoides), A0AOP9FAF0 (Kouleothrix aurantiaca), A4WW35 } \\
\text { (Rhodobacter sphaeroides), P04718 (Rhodospirillum rubrum), } \\
\text { Q12TQ0 (Methanococcoides burtonii), A0A1L3Q3Y6 } \\
\text { (Methanohalophilus halophilus), B5IH56 (Aciduliprofundum } \\
\text { boonei), O93627 (Thermococcus kodakarensis), J1ANE7 } \\
\text { (Methanofollis liminatans), and Q2FSY4 (Methanospirillum } \\
\text { hungatei). The sequences for representative Form I' homologs } \\
\text { are presented in this study (Supplementary Data 1). b, Overlay } \\
\text { of amino acid residues } 408-458 \text { of Syn6301 Rubisco (tan) with } \\
\text { residues } 415-453 \text { of P. breve Rubisco (blue) depicting the } \\
\text { unique RbcL C-terminal extension found in Form I enzymes, } \\
\text { but not in Rubisco homologs that do not possess RbcS. } \\
\text { Residues R428, N429, and E430 of Syn6301 RbcL contact } \\
\text { residues N29 and Y32 at the interface of Syn6301 RbcS } \\
\text { (purple). }\end{array}$ \\
\hline Extended Data Fig. 5 & $\begin{array}{l}\text { Negative-staining } \\
\text { electron microscopy 2D } \\
\text { images of P. breve } \\
\text { Rubisco. }\end{array}$ & Shih_EDFig5.eps & $\begin{array}{l}\text { Images reflect the highest resolution data collected with } \\
\text { activated P. breve Rubisco in phosphate buffer. The } \\
\text { experiment was performed once }(n=1) \text {. }\end{array}$ \\
\hline Extended Data Fig. 6 & $\begin{array}{l}\text { Extended SEC-SAXS- } \\
\text { MALS data. }\end{array}$ & Shih_EDFig6.eps & $\begin{array}{l}\text { Experimental SAXS profiles (black) of P. breve Rubisco in the } \\
\text { absence (purple) or presence (blue) of bound } 2 \text { CABP is }\end{array}$ \\
\hline
\end{tabular}




\begin{tabular}{|c|c|c|c|}
\hline & & & $\begin{array}{l}\text { displayed with the calculated scattering from the atomistic } \\
\text { models shown in Fig. } 3 c \text {. Inset shows the Guinier plot of } \\
\text { experimental SAXS profiles with the linear fit in the } q \times R g<1.6 \\
\text { limits. }\end{array}$ \\
\hline Extended Data Fig. 7 & $\begin{array}{l}\text { Amino acid sequence } \\
\text { alignment of Syn } 6301 \\
\text { RbcL and P. breve RbcL. }\end{array}$ & Shih_EDFig7.eps & $\begin{array}{l}\text { a, Structure-based sequence alignment was originally made } \\
\text { using PROMALS3D }{ }^{53} \text { using } 1 R B L \text { and } 6 U R A \text { structures, then } \\
\text { aligned with the complete RbcL sequences using MAFFT }{ }^{54} \text {. } \\
\text { Darker shades indicate higher sequence conservation } \\
\text { between amino acids. Syn6301 and P. breve RbcL residues } \\
\text { involved in dimer-dimer interactions are highlighted in green } \\
\text { and blue, respectively. Syn6301 RbcL residues involved in RbcS } \\
\text { contacts are annotated with red stars. All contact residues } \\
\text { were identified using CCP4 CONTACTS } \text {. b-c, Cross-section }^{55} \text { depictions of } 1 \text { RBL, without RbcS, and P. breve Rubisco } \\
\text { highlighting dimer-dimer interactions as in panel a.d, Map of } \\
\text { Syn6301 RbcL residues involved in RbcS interactions, } \\
\text { highlighted in red as in panel a. }\end{array}$ \\
\hline Extended Data Fig. 8 & $\begin{array}{l}\text { Mutating key amino } \\
\text { acid residues at the } \\
\text { dimer-dimer interface } \\
\text { of } P \text {. breve Rubisco } \\
\text { disrupts octameric } \\
\text { oligomeric assembly. }\end{array}$ & Shih_EDFig8.eps & $\begin{array}{l}\text { Native PAGE gel of recombinant WT, K150A, D161A, W165A, } \\
\text { D220A, and Y224A P. breve Rubisco. Native Mark protein } \\
\text { ladder denoted by “M". Site directed mutants destabilize the } \\
\text { interface between RbcL dimers leading to break down of } \\
\text { higher-order (i.e., } \mathrm{L}_{8} \text { ) oligomers into Rubisco species with } \\
\text { variable oligomeric state and conformations, which results in a } \\
\text { variety of lower molecular weight migration patterns within } \\
\text { the Native PAGE gel. Experiment was performed once }(n=1) \text {. }\end{array}$ \\
\hline Extended Data Fig. 9 & $\begin{array}{l}\text { Site directed } \\
\text { mutagenesis of Syn6301 } \\
\text { dimer-dimer interface } \\
\text { residues imparts } \\
\text { marginal stability in the } \\
\text { absence of RbcS. }\end{array}$ & Shih_EDFig9.eps & $\begin{array}{l}\text { a, Protein thermal shift data displaying the mean fluorescent } \\
\text { signal collected from four separate trials for WT Syn6301 RbcL, } \\
\text { three separate mutant proteins, L158W, V154D, D349R and a } \\
\text { combined four mutant protein, 4SDM (L158W, V154D, F217Y, } \\
\text { and D349R). Mutations were designed to reflect homologous } \\
\text { dimer-dimer interface residues present in P. breve Rubisco. } \\
\text { The peaks corresponding to thermal denaturation of } \mathrm{L}_{8} \\
\text { quaternary structure are boxed, and analysis statistics are } \\
\text { presented in the below table. Tm values represent the mean }\end{array}$ \\
\hline
\end{tabular}




\begin{tabular}{|l|l|l|}
\hline & & \\
& & $\begin{array}{l}\text { and standard deviation of } n \text { number of experiments } \\
\text { conducted with the same protein sample. Two-tailed P-values } \\
\text { for unpaired t test with Welch's corrections are reported in } \\
\text { the last column using WT Syn6301 RbcL as the reference } \\
\text { comparison. } n=\text { number of technical replicates conducted in } \\
\text { experiment. ns }=\text { not significant. } * * P<0.005, * * * P<0.0005 . \\
\text { b, Native gel of purified recombinant WT and mutant Syn6301 } \\
\text { proteins used in experiment. }\end{array}$ \\
\hline
\end{tabular}

2. Supplementary Information:

5

\begin{tabular}{|l|l|l|l|}
\hline Item & Present? & $\begin{array}{l}\text { Filename } \\
\text { This should be the name } \\
\text { the file is saved as when it } \\
\text { is uploaded to our system, } \\
\text { and should include the file } \\
\text { extension. The extension } \\
\text { must be .pdf }\end{array}$ & $\begin{array}{l}\text { A brief, numerical description of file contents. } \\
\text { i.e.: Supplementary Figures 1-4, Supplementary Discussion, and }\end{array}$ \\
\hline Supplementary Information & Yes & $\begin{array}{l}\text { Supplementary } \\
\text { Information.pdf }\end{array}$ & Supplementary Note, Supplementary Tables 1-3. \\
\hline Reporting Summary & Yes & $\begin{array}{l}\text { nr-reporting- } \\
\text { summary_PMS_2020071 } \\
0 . p d f\end{array}$ & \\
\hline
\end{tabular}




\begin{tabular}{|c|c|c|c|}
\hline Type & $\begin{array}{l}\text { Number } \\
\text { If there are multiple files of the same } \\
\text { type this should be the numerical } \\
\text { indicator. i.e. "1" for Video 1, "2" for } \\
\text { Video 2, etc. }\end{array}$ & $\begin{array}{l}\text { Filename } \\
\text { This should be the name the file is } \\
\text { saved as when it is uploaded to our } \\
\text { system, and should include the file } \\
\text { extension. i.e.: Smith } \\
\text { Supplementary Video 1.mov }\end{array}$ & $\begin{array}{l}\text { Legend or Descriptive Caption } \\
\text { Describe the contents of the file }\end{array}$ \\
\hline Supplementary Data & 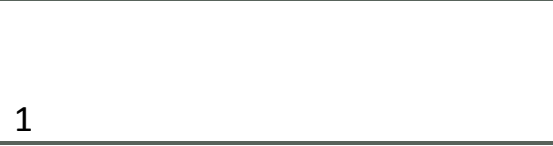 & Supp. Data 1.txt & $\begin{array}{l}\text { Fasta file containing protein } \\
\text { amino acid sequences for Form I' } \\
\text { enzymes identified from MAGs. }\end{array}$ \\
\hline Supplementary Data & 2 & Supp. Data 2.xlsx & $\begin{array}{l}\text { Representative MAG genbank } \\
\text { scaffolds. }\end{array}$ \\
\hline Supplementary Data & 3 & Supp. Data 3.xlsx & $\begin{array}{l}\text { Site-directed mutagenesis primers } \\
\text { and synthesized candidate Form I' } \\
r b c L \text { gene sequences. }\end{array}$ \\
\hline
\end{tabular}




\section{Novel Bacterial Clade Reveals Origin of Form I Rubisco}

Douglas M. Banda ${ }^{1,2}$, Jose H. Pereira ${ }^{3,4, \dagger}$, Albert K. Liu ${ }^{1,2, \dagger}$, Douglas J. Orr ${ }^{5}$, Michal Hammel ${ }^{4}$, Christine $\mathrm{He}^{6}$, Martin A.J. Parry ${ }^{5}$, Elizabete Carmo-Silva ${ }^{5}$, Paul D. Adams ${ }^{3,4}$, Jillian F. Banfield $^{6,7,8,9, *} \&$ Patrick M. Shih ${ }^{1,2,10,11, *}$

${ }^{1}$ Department of Plant Biology, University of California, Davis, CA, USA.

${ }^{2}$ Environmental Genomics and Systems Biology Division, Lawrence Berkeley National

${ }^{3}$ Technology Division, Joint BioEnergy Institute, Emeryville, CA, USA.

${ }^{4}$ Molecular Biophysics and Integrated Bioimaging Division, Lawrence Berkeley National USA.

${ }^{7}$ Department of Environmental Science, Policy, and Management, University of California, Berkeley, Berkeley, CA, USA.

${ }^{10}$ Feedstocks Division, Joint BioEnergy Institute, Emeryville, CA, USA.

${ }^{11}$ Genome Center, University of California, Davis, Davis, CA, USA.

${ }^{\dagger}$ Contributed equally

41

*Correspondence to: pmshih@ucdavis.edu or jbanfield@berkeley.edu

\section{Abstract}

Rubisco sustains the biosphere through the fixation of $\mathrm{CO}_{2}$ into biomass. In plants and cyanobacteria, Form I Rubisco is structurally comprised of large and small subunits, whereas all other Rubisco Forms lack small subunits. Thus, the rise of the Form I complex through the innovation of small subunits represents a key, yet poorly understood, transition in Rubisco's evolution. Through metagenomic analyses, we discovered a previously uncharacterized clade sister to Form I Rubisco that evolved without small subunits. This clade diverged prior to the evolution of cyanobacteria and the origin of the small subunit; thus, it provides a unique reference point to advance our understanding of Form I Rubisco evolution. Structural and kinetic data presented here reveal how a proto-Form I Rubisco assembled and functioned without the 
53 structural stability imparted from small subunits. Our findings provide insight into a key

54 evolutionary transition of the most abundant enzyme on Earth and the predominant entry point

55 for nearly all global organic carbon.

\section{Main Text:}

57 Of all known enzymes, few have been more integral in linking the evolution of life with 58 the geochemical cycles of our planet than Rubisco (D-ribulose 1,5-bisphosphate 59 carboxylase/oxygenase $)^{1}$. Rubisco sources nearly all organic carbon to the biosphere through the 60 fixation of atmospheric $\mathrm{CO}_{2}$ with ribulose 1,5-bisphosphate (RuBP) into biomass, thus sustaining

61 our entire food supply. Rubisco also possesses competing oxygenase activity, which is thought to

62 be a vestige of its evolution in a young, oxygen-depleted atmosphere; yet it has co-evolved with

63 Rubisco's carboxylase activity over billions of years. Although there are several distinct Forms

64 of Rubisco found across all three domains of 1 life ${ }^{2,3}$, the vast majority of carbon fixation on Earth

65 is driven specifically by Form I Rubisco (found in plants, cyanobacteria, algae, and select

66 bacteria phyla); thus, the evolution of this unique Form of Rubisco has profoundly shaped the

67 trajectory of our planet.

$69 \mathrm{kDa}$ ) which assemble head-to-tail as catalytically active dimers. From this rudimentary dimeric

70 scaffold (found in Form II and III homologs), Rubisco has evolved to function in higher-order

71 structures of large subunits including hexamers (Form II), octamers (Form I), and decamers

72 (Form III). Form I homologs, however, are structurally unique from their divergent Form II and

73 Form III counterparts due to the presence of additional small subunits ( $\mathrm{RbcS}, \sim 13-17 \mathrm{kDa}$ ),

74 which cap either end of a central octameric RbcL assembly to form a hexadecameric $\left(\mathrm{L}_{8} \mathrm{~S}_{8}\right)$

75 holoenzyme. Thus, understanding the origins of $\mathrm{RbcS}$ is part and parcel to investigating the

76 evolution of Form I Rubisco. 
Although not in direct participation with the active site, RbcS is accepted as an

78 indispensable structural component of Form I Rubisco ${ }^{4-6}$. For example, cyanobacterial Rubisco

79 from Synechococcus sp. strain PCC 6301 (Syn6301) retains approximately 1\% of its carboxylase

80 activity in the absence of $\mathrm{RbcS}^{4}$, suggesting that active site structural integrity is compromised.

81 Furthermore, Form I Rubisco from Rhodobacter sphaeroides relies on RbcS to correctly arrange

82 RbcL geometry for proper activity ${ }^{7}$, and plant Rubisco RbcL form insoluble aggregates when

83 expressed without RbcS in planta $^{8,9}$. Despite its demonstrated significance in Rubisco catalysis,

84 the structural role RbcS has played in the evolution of Form I Rubisco has long been debated ${ }^{6}$.

85 This quandary, in part, stems from the fact that we have not identified Form I Rubisco that

86 function without small subunits. Thus, the identification and characterization of a small subunit-

87 less Form I Rubisco would provide the necessary reference point from which to better examine

88 the evolutionary role of RbcS. Towards this end, we searched metagenomic datasets for a

89 "missing link" between the evolution of the Form I clade and all other Forms of Rubisco. Here,

90 we report the discovery of a Form I Rubisco with octameric oligomeric assembly that evolved

91 without $\mathrm{RbcS}$, thus challenging our understanding of the structural properties that govern the

92 activity of the most prominent Form of Rubisco.

93 Discovery of Form I Rubisco that lack small subunits

94 To determine whether Form I Rubisco lacking small subunits occur in nature, we

95 analyzed a diverse set of metagenomic datasets derived from environmental communities of 96 largely uncultivated bacteria. Our analyses specifically targeted the identification of

97 uncharacterized bacterial $r b c L$ genes, which are usually found within operons encoding other key

98 Calvin-Benson-Bassham (CBB) cycle genes ${ }^{10}$. Through this process, we identified $24 \mathrm{rbcL}$ genes

99 with gene products that share high sequence homology (52-61\%) to known Form I Rubisco. 
100 Notably, the average amino acid sequence identity between different Forms of Rubisco is 101 approximately $30 \%$, thus it is possible that the identified $r b c L$ genes were either within the Form

102 I clade, or within a close sister clade ${ }^{2}$. Further phylogenetic analyses confirmed that the newly 103 discovered $r b c L$ sequences indeed form a monophyletic clade sister to Form I Rubisco. Given 104 the unique phylogenetic proximity to Form I, we named this new clade Form I' to distinguish it 105 from all other bona fide Forms of Rubisco (Fig. 1a).

Where metagenome-assembled contigs were of sufficient length to reveal the genomic context surrounding Form I' rbcL genes, all identified operons encoded other CBB cycle genes, 108 including the only other CBB cycle-specific gene, phosphoribulokinase (PRK) (Fig. 1b). Closer 109 inspection of metagenome-assembled genomes (MAGs) containing Form I' rbcL genes indicated 110 the absence of $r b c S$ upstream or downstream of $r b c L$. Notably, bacterial Form I $r b c L$ and $r b c S$ 111 genes are always found within one or two genes of another in operons ${ }^{11,12}$. Given that Form I' 112 Rubisco lacks RbcS similar to all other non-Form I Rubisco found in various bacteria and 113 archaea, this suggests that the Form I' clade represents a distinct Form of Rubisco that likely 114 diverged from the Form I clade prior to the origin of $\mathrm{RbcS}$.

Surprisingly, all Form I' genes identified from MAGs were found exclusively in a single order of the Chloroflexi phylum, Anaerolineales (Extended Data Figs. 1 and 2). Although

117 Chloroflexi are commonly known for their phototrophic members in the order Chloroflexales, 118 the majority of the phylum is composed of phenotypically-diverse filamentous bacteria that are 119 non-phototrophic, such as the Anaerolineales ${ }^{13}$. Of the known phototrophic examples of 120 Chloroflexi within the order Chloroflexales, most perform carbon fixation via the 3121 hydroxypropionate bicycle (e.g. Chloroflexus sp.), or with Form I Rubisco via the CBB cycle 122 (e.g. Oscillochloris trichoides, Chlorothrix halophila, and Kouleothrix aurantiaca) ${ }^{14}$. Form I'- 
123 containing MAGs were not found to contain characteristic 3HP bicycle genes such as propionyl-

124 CoA synthetase, malonyl-CoA reductase/3-hydroxypropionate dehydrogenase, and malonyl-

$125 \mathrm{CoA} /$ succinyl-CoA reductase, suggesting that the bacteria consortium from which MAGs were

126 derived use the CBB cycle for autotrophy. Though some examples of phototrophic Chloroflexi

127 have recently been described in clades sister to the Anaerolinea (e.g. the class-level clade

128 Candidatus Thermofonsia) ${ }^{15}$, none possessed carbon fixation pathway genes and were presumed 129 to be photoheterotrophic. Studies have demonstrated that phototrophy within Chloroflexi may be

130 driven by horizontal gene transfer ${ }^{15,16}$; however, the tight phylogenetic distribution of Form I' 131 genes within the order Anaerolineales suggests otherwise, albeit future studies may reveal 132 genomes outside of Anaerolineales that possess Form I' genes.

\section{Form I' Rubisco is functional despite lack of small subunits}

To characterize genes discovered from MAGs, representative Form I' Rubisco homologs

135 were recombinantly expressed and purified (Extended Data Fig. 3a) from E. coli

136 overexpressing the bacterial chaperonin system GroEL-GroES (homologous to Cpn60-Cpn10/20

137 in plants), a necessary component of Rubisco biogenesis ${ }^{17,18}$. The assembly of hexadecameric

138 Form I homologs in cyanobacteria and plants require auxiliary chaperones such as RbcX and

139 Raf1, which aid in the stabilization of the octameric RbcL core before the addition of small

140 subunits ${ }^{19,20}$. Other Form I homologs, however, do not require homologous assembly factors but

141 instead rely on RbcS for efficient assembly, which has been demonstrated for Rubisco from the

142 photosynthetic proteobacterium Rhodobacter sphaeroides ${ }^{7}$. RbcX was not found in Form I'

143 containing MAGs (Fig. 1b). Consistent with this finding, all Form I' sequences do not possess

144 the C-terminal binding domain for $\mathrm{RbcX}^{8,21}$ (Extended Data Fig. 4a). Furthermore, Form I'

145 homologs identified to date do not possess small subunits, precluding the necessity of chaperones 
146 involved in the assembly of hexadecameric Rubisco ${ }^{19}$. Some archaeal Rubisco possess an extra

147 C-terminal domain that is proposed to aid in $\mathrm{RbcL}$ core assembly ${ }^{22}$, but this unique insertion is 148 not found within the described representative homologs of the Form I' clade (Extended Data

149 Fig. 4a). Notably, Syn6301 Rubisco expressed in E. coli makes up $\sim 1-2 \%$ of the total soluble 150 protein, but this number improves to $\sim 6 \%$ with the associated overexpression of GroEL/ES ${ }^{23}$. In

151 comparison, Rubisco from $R$. sphaeroides comprises $\sim 16 \%$ of the total soluble protein when 152 heterologously expressed in E. coli, which jumps to $33 \%$ with the overexpression of GroEL/ES ${ }^{7}$.

153 With the system outlined in this work, Form I' Rubisco was found to express at $\sim 7-8 \%$ of the 154 total soluble protein in BL21(DE3) E. coli, which improves to $\sim 14-15 \%$ when overexpressed 155 with GroEL/ES (Extended Data Fig. 3b). Currently, it is unknown whether the expression 156 levels of Form I' Rubisco in E. coli are intrinsic to its amino acid sequence alone, or if auxiliary 157 chaperone factors are necessary for higher expression. Though the Chloroflexi from which these 158 sequences are derived may possess a unique assembly factor that aids in Rubisco biogenesis, no 159 such protein was identified from the metagenomic datasets presented in this work.

To assess the catalytic activity of a representative Form I' homolog, we performed 161 detailed enzyme kinetic measurements on Form I' Rubisco from the mesophilic Chloroflexi 162 species "Candidatus Promineofilum breve" (P. breve) using the method of Parry et al. ${ }^{24}$. At 163 saturating substrate concentrations, Rubisco proteins exhibit maximal rates of catalysis $\left(V_{C}\right.$ and $164 V_{O}$ for carboxylation and oxygenation, respectively), generally at the expense of the 165 concentration of substrate necessary to achieve a maximal rate (represented by the Michaelis 166 constants $K_{C}$ and $K_{O}$ for carboxylation and oxygenation, respectively, which can be considered 167 conceptually as pseudo-dissociation constants for the binding of either $\mathrm{CO}_{2}$ or $\left.\mathrm{O}_{2}\right)^{25,26}$. 
169 to the reported measurements of Form I enzymes at $25{ }^{\circ} \mathrm{C}^{26}$ (Table 1, Fig. 2). Conversely, the

170 enzyme demonstrated slightly above average $V_{O}$ and below average $K_{O}$. This is consistent with

171 the discovery of the Form I' clade within the order Anaerolineales, which is typically comprised

172 of obligate anaerobes ${ }^{27}$, although genomic signatures of aerobic respiration have recently been

173 discovered in some examples of Anaerolineae ${ }^{28,29}$. Together, these kinetic parameters culminated

174 in a specificity for $\mathrm{CO}_{2}$ over $\mathrm{O}_{2}$ (represented by the specificity factor $\left(S_{C / O}\right)$, a measure of the

175 catalytic efficiency of the carboxylation reaction over the oxygenation reaction) that is lower

176 relative to values reported for Form I enzymes, but higher than Form II and Form III homologs

177 (Supplementary Table 1). It is unclear at this time whether the high oxygenase specificity of P.

178 breve Rubisco is linked to the absence of RbcS. Notably, Form I' and Form I Rubisco lineages

179 diverged before the evolution of cyanobacteria suggesting that Form I' enzymes may have

180 evolved in anaerobic conditions.

\section{Form I' Rubisco is octameric, reminiscent of Form I Rubisco}

The Form I clade is structurally characterized by two features distinct from other Forms

183 of Rubisco: 1) the presence of $\mathrm{RbcS}$, and 2) the oligomeric assembly of RbcL into octamers.

184 Given the close phylogenetic placement to the Form I clade, we hypothesized that Form I'

185 homologs may possess octameric oligomeric assembly of RbcL, which has not been previously

186 observed for Rubisco in nature. Size exclusion chromatography (SEC) and non-denaturing

187 PAGE analyses revealed that recombinant P. breve RbcL dimers ( $100-110 \mathrm{kDa})$ oligomerized

188 into a higher-order structure (Fig. 3d). Previous studies have demonstrated that the addition of

189 the Rubisco-specific transition-state analog, 2-carboxyarabinitol 1,5-bisphosphate (2CABP), may

190 influence the oligomeric state of the enzyme ${ }^{30}$. Incubation of magnesium-bound and $\mathrm{CO}_{2}$ - 
191 activated P. breve Rubisco with 2CABP resulted in an observed structural compaction, evident 192 from both later elution in SEC traces, as well as slower migration in non-denaturing gels (Fig. 3).

194 Rubisco, we performed SEC coupled to small-angle X-ray scattering (SAXS) and multiangle light scattering (MALS) (SEC-SAXS-MALS) experiments ${ }^{31}$ with activated P. breve Rubisco in 196 the presence or absence of 2CABP. Protein molecular weights determined by MALS ( 400-440 $197 \mathrm{kDa}$ ) supported the oligomerization of $\mathrm{P}$. breve Rubisco as an $\mathrm{L}_{8}$ complex (theoretical octamer 198 M.W. $\sim 409 \mathrm{kDa}$ ), similar to the octameric assembly of RbcL in related Form I enzymes (Fig. 3). 199 These observations were corroborated by negative-staining electron microscopy images 200 (Extended Data Fig. 5). Experimentally determined pair-distribution, or P(r), functions 201 displayed significant broadening and elongation of P. breve Rubisco in the absence of 2CABP 202 relative to the $2 \mathrm{CABP}-$ bound protein (Fig. 3b). This observation agrees well with the larger 203 radius of gyration $(\mathrm{Rg})$ values of the $2 \mathrm{CABP}-$ bound $(\mathrm{Rg} \sim 46.8 \pm 0.4 \AA)$ versus unbound $(\mathrm{Rg} \sim$ $204 \quad 45.0 \pm 0.5 \AA$ ) protein.

In the absence of substrate, Form I Rubisco proteins exist in an "open" conformation that

206 is structurally characterized, in part, by an extended C-terminal domain that is disordered and 207 positioned away from the active $\operatorname{site}^{32}$. Upon active site binding of RuBP, the extended C208 terminal domain flips down over the active site with Loop 6 to produce a compact "closed" 209 conformation primed for catalysis. In order to account for observed differences in the radius of 210 gyration between 2CABP-bound and unbound structures, we generated theoretical SAXS data 211 from computational models of octameric P. breve Rubisco either in a compact "closed" state 212 (i.e., bound to 2CABP) or an "open" state with disordered C-terminal domains (Fig. 3c). Indeed, 213 theoretical SAXS data produced from these models matched well with the experimentally 
214 determined P(r) functions (Fig. 3b) and SAXS profiles (Extended Data Fig. 6, Supplementary

215 Table 2, $\quad{ }^{2}=1.8$ and 1.4 for closed and open models, respectively).

Overall, the combination of SEC-SAXS-MALS and electron microscopy experiments

217 support an $\mathrm{L}_{8}$ oligomerization of Form I' Rubisco reminiscent of the $\mathrm{L}_{8} \mathrm{~S}_{8}$ Form I Rubisco.

218 Because no other Form of Rubisco has been convincingly demonstrated to express as octamers in

219 nature (see Supplementary Note), the most parsimonious history consistent with our data

220 suggests that the common ancestor of Form I and Form I' clades evolved an octameric core

221 assembly prior to the evolution of RbcS.

222 Form I' Rubisco structure yields insight into Form I Rubisco evolution

223 To obtain higher molecular resolution of P. breve Rubisco, we solved a $2.2 \AA$ crystal

224 structure of the activated enzyme in complex with 2CABP (Fig. 4, Supplementary Table 3).

225 Superposition of P. breve RbcL onto the structure of Syn6301 $\mathrm{L}_{8} \mathrm{~S}_{8}$ Rubisco (PDB ID: 1RBL) ${ }^{33}$

226 resulted in a C $\alpha$ RMSD of $0.68 \AA$ between 435 pruned atom pairs $(97.5 \%$ of P. breve RbcL

227 amino acid sequence), with a Q-score of $0.87^{34}$. As with all other bona fide Rubisco, all key

228 active site residues ${ }^{35,36}$ were positioned in an $\alpha \beta$-barrel (TIM-barrel) domain (residues 158-405).

Many of the characteristic Form I hydrophobic RbcL residues at the interface of large and 230 small subunits ${ }^{37}$ were either functionally substituted on the surface of P. breve Rubisco $(\sim 31 \%)$

231 or completely absent ( 4\%), based on sequence homology to Syn6301 RbcL (Extended data

232 Fig. 7). RbcL surface residues between the two structures displayed strikingly similar

233 electrostatic characteristics (Fig. 4), which was unexpected given that P. breve Rubisco had not

234 evolved to interact with RbcS, unlike its closely related Syn6301 homolog. Because of this 235 observation and the close phylogenetic relationship between the Form I and Form I' clades, a 
236 competing hypothesis is that Form I' evolved from Form I homologs and subsequently lost

237 RbcS, as opposed to the hypothesis that Form I' and Form I Rubisco diverged from a common

238 ancestor. To explore this further, we investigated the observation that Form I homologs possess

239 an RbcL "C-terminal extension" (residues 430-442 of Syn6301 Rubisco, Extended Data Fig.

240 4a) not found in Rubisco that lack RbcS (i.e., all other Forms of Rubisco). This unique C-

241 terminal extension has evolved in Form I lineages to stabilize key RbcL interactions with $\mathrm{RbcS}^{38}$

242 (Extended Data Fig. 4b). The Form I' enzymes identified in this study do not possess this

243 unique C-terminal extension important for $\mathrm{RbcS}$ interactions, supporting the hypothesis that

244 Form I' and Form I Rubisco diverged from a common ancestor. This is in accordance with the

245 parsimonious observation that all non-Form I Rubisco lack RbcS, suggesting that the common

246 ancestor to both Form I and Form 1' clades most likely lacked RbcS.

In the absence of RbcS, we hypothesized that P. breve Rubisco must possess fortified

248 interactions at the RbcL dimer-dimer interface to support octameric assembly. Indeed, P. breve

249 Rubisco possesses an extensive network of hydrogen bonds and salt bridges at the interdimer

250 interface that is not present in Syn6301 Rubisco (Fig. 5a). Site-directed mutagenesis of key

251 amino acid residues within this network (Lys150, Asp161, Trp165, Asp220, and Tyr224) to

252 alanine abolished P. breve Rubisco's octameric assembly (Extended Data Fig. 8),

253 demonstrating their importance in maintaining holoenzyme stability in the absence of RbcS.

254 Notably, homologous amino acid positions to Asp161, Trp165, and Tyr224 within Syn6301

255 (Val154, Leu158, and Phe217, respectively) are incapable of forming a similar electrostatic

256 network due to their side-chain physicochemical properties, necessitating interactions with RbcS

257 for complex stability (Extended Data Fig. 7). 
259 affect the thermal stability of the complex quaternary structure, we employed a protein thermal

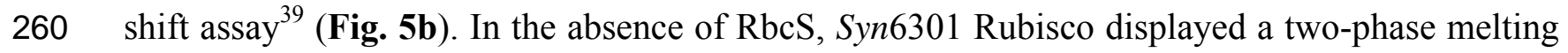
261 profile; the first phase $\left(\mathrm{Tm}=58.6 \pm 0.2^{\circ} \mathrm{C}\right)$ resulting from quaternary structure disassembly (i.e., 262 the dissociation of octamers into dimers), and the second phase $\left(\mathrm{Tm}=70.6 \pm 0.2{ }^{\circ} \mathrm{C}\right)$ 263 corresponding to the simultaneous denaturation of RbcL dimers and RbcL secondary structure ${ }^{40}$. 264 In the presence of RbcS, Syn6301 Rubisco was significantly stabilized such that $\mathrm{L}_{8} \mathrm{~S}_{8}$ 265 disassembly was shifted by more than $15{ }^{\circ} \mathrm{C}$ relative to Syn6301 $\mathrm{L}_{8}\left(\mathrm{Tm}=75.5 \pm 0.1{ }^{\circ} \mathrm{C}\right)$. 266 Interestingly, P. breve Rubisco disassembly displayed a modest increase in $\mathrm{Tm}\left(82.6 \pm 0.1{ }^{\circ} \mathrm{C}\right)$ 267 relative to Syn6301 $\mathrm{L}_{8} \mathrm{~S}_{8}$, but a significant increase when compared to the Tm measured for 268 Syn6301 in the absence of RbcS, consistent with the predicted added stability due to interdimer 269 interface interactions. To stabilize Syn6301 in the absence of RbcS, we mutated RbcL residues 270 known to interact with $\mathrm{RbcS}$ to mimic part of the electrostatic network stabilizing P. breve 271 oligomeric assembly (Extended Data Fig. 9). This effort yielded modest improvement in 272 stability, highlighting the complexity of forming octamers in the absence of RbcS.

\section{Discussion}

274

275 Accrued evidence from investigations into the evolutionary adaptability of proteins supports a 276 common trend: the catalytic promiscuity of an enzyme is inversely proportional to its 277 conformational stability ${ }^{41-43}$. In line with previous observations ${ }^{6}$, the data presented in this work 278 suggests that the innovation of a distinct structural subunit (i.e., RbcS) imparted structural 279 stability to Rubisco during the evolution of its carboxylase and oxygenase activities towards 280 "Pareto optimality",44. Form I' Rubisco from Ca. P. breve demonstrated high oxygenase activity 
281 and lower specificity when compared to Form I homologs (Fig. 2, Table 1), likely stemming 282 from the anaerobic lifestyle of the Anaerolineales order of Chloroflexi from which sequences 283 were discovered. Furthermore, the divergence of Form I' and Form I Rubisco from a common 284 ancestor predates the origin of cyanobacteria; thus it is likely that Form I' Rubisco originated 285 during the Archean Eon when atmospheric oxygen was scarce. Collectively, these observations 286 suggest that the appearance of $\mathrm{RbcS}$ and the evolutionary transition from $\mathrm{L}_{8}$ to $\mathrm{L}_{8} \mathrm{~S}_{8}$ may have 287 been an evolutionary response to the rise of oxygen $\sim 2.4 \mathrm{Ga}$. This environmental transition may 288 have provided a strong selective pressure to $\mathrm{L}_{8}$-containing autotrophs (e.g., stem-group 289 cyanobacteria) that necessitated a tradeoff between conformational rigidity (i.e., enhanced 290 interactions at the dimer-dimer interface of octameric Rubisco) and active site plasticity. The 291 selective pressure driving this tradeoff likely stemmed from an increased demand for improved 292 carboxylation activity to drive flux through carbon metabolism during a rapidly changing 293 paleoatmosphere ${ }^{45,46}$. To evolve this conformational dynamism while maintaining an optimized 294 oligomeric state (i.e., $\mathrm{L}_{8}$ ), we posit that $\mathrm{RbcS}$ evolved to facilitate the adaptive evolution of 295 Rubisco's catalytic activity, effectively buffering the cost of destabilizing mutations and 296 allowing the sampling of higher genetic diversity during the random walk through sequence 297 space.

In addition to the evolutionary insight gleaned from this work, the discovery of the Form 299 I' clade from MAG's may offer alternative means to explore Rubisco engineering efforts in 300 plants. Notably, Form I Rubisco has long been recalcitrant to directed evolution experiments for 301 improved carbon fixation, with notable exceptions ${ }^{47}$, in part due to challenges associated with 302 effectively exploring the sequence space of two genes (i.e., RbcL and RbcS) simultaneously; 303 thus, the absence of RbcS in Form I' enzymes may streamline such future efforts. Overall, 
304 performing directed evolution experiments ${ }^{47,48}$ with P. breve Rubisco in conjunction with the 305 continued characterization of the Form I' clade will offer novel opportunities to advance our understanding

of Rubisco evolution.

\section{Methods}

Metagenomic and phylogenetic analysis. All metagenomes were sequenced using $150 \mathrm{bp}$, paired-end Illumina reads and assembled into scaffolds using either IDBA-UD or Megahit. Scaffolds were binned based on GC content, coverage, presence of ribosomal proteins, presence/copies of single copy genes, tetranucleotide frequency, and patterns of coverage across samples. Bins were manually curated, dereplicated, and filtered for completeness and contamination. Genes were predicted using hidden Markov models (HMMs) based on Pfam, TIGRfams, KEGG, and custom databases. Phylogeny of bins containing Rubisco genes was identified using overall scaffold gene content as well as maximum likelihood phylogenetic trees of 16 concatenated ribosomal protein sequences. Rubisco gene sequences were dereplicated at 97\% amino acid identity using CD-Hit, aligned using MAFFT (default parameters), and columns with $>95 \%$ gaps were removed using TrimAI. A maximum-likelihood phylogenetic tree was constructed using RAxML-HPC BlackBox (v. 8.2.10) as implemented on cipres.org (default parameters with LG model). To construct Figure 1A, branches with bootstrap values of $<0.65$ were collapsed. Both the alignment file and the tree file with bootstrap values are available on figshare (DOI: 10.6084/m9.figshare.9980630).

Plasmids, cloning, and site-directed mutagenesis. Representative Form I' rbcL genes were synthesized by Twist Biosciences (San Francisco, CA) (sequences available as supplementary data) and cloned into a pET28 vector with an N-terminal His s $_{14}$-bdSUMO tag ${ }^{59}$. Plasmids pSF1389 ${ }^{59}$, pET11a-Syn6301-rbcLS, pET11A-Syn6301-rbcL, pBADES/EL, and pG-KJE8 ${ }^{21}$ were gifts. Site-directed mutagenesis (SDM) was conducted using an Agilent QuikChange SDM kit and standard procedures. Primers were designed using the Agilent QuikChange Primer Design tool (available as supplementary data).

Expression and purification of recombinant proteins. Brachypodium distachyon SUMOspecific protease (bdSENP1) was prepared by transforming pSF1389 into chemically competent 
BL21 DE3 Star E. coli cells (Macrolab, QB3-Berkeley, CA). Cells were grown to mid-log phase at $37{ }^{\circ} \mathrm{C}\left(\mathrm{OD}_{600} \sim 0.6\right)$ and induced with $0.3 \mathrm{mM}$ IPTG for 3 hours. Cells were resuspended in pH 7.0 Lysis Buffer (20 mM sodium phosphate, $300 \mathrm{mM} \mathrm{NaCl}, 10 \mathrm{mM}$ imidazole, 5\% glycerol, $2 \mathrm{mM} \mathrm{MgCl}_{2}$ ) with $\sim 5 \mathrm{mM}$ PMSF and subject to a freeze-thaw cycle before lysis by use of a Microfluidizer high pressure homogenizer (Microfluidics, Westwood, MA), and centrifugation $(15,000 \mathrm{RCF}, 20 \mathrm{~min})$. Soluble protein was $0.2 / 0.8 \mu \mathrm{m}$ filtered and applied to Ni-NTA Resin (Thermo Fisher, Waltham, MA) and batch bound according to the manufacturer's protocols. Columns were washed thoroughly before elution. TEV protease (MilliporeSigma, Burlington, MA) was added to the eluted fraction according to the manufacturer's suggestion and rocked gently overnight at $4{ }^{\circ} \mathrm{C}$ to facilitate His tag cleavage. The flow-through from TEV protease reactions was buffer exchanged into $\mathrm{pH} 7.0 \mathrm{Ni}$ Equilibration buffer ( $20 \mathrm{mM}$ sodium phosphate, $300 \mathrm{mM} \mathrm{NaCl}, 10 \mathrm{mM}$ imidazole, 10\% glycerol) and passed over Ni-NTA resin again to separate cleaved His tag from the target protein. bdSENP1-containing flow-through was analyzed by SDS-PAGE for purity and stored at $-80{ }^{\circ} \mathrm{C}$ in storage buffer $(20 \mathrm{mM}$ sodium phosphate $\mathrm{pH}$ 7.0, $300 \mathrm{mM} \mathrm{NaCl}, 1 \mathrm{mM}$ DTT, 10\% glycerol).

P. breve Rubisco was prepared by co-transforming plasmids containing His 14 -bdSUMOtagged P. breve RbcL into chemically competent BL21 DE3 Star E. coli with pBADES/EL plasmid. Cells were grown to mid-log phase at $30{ }^{\circ} \mathrm{C}\left(\mathrm{OD}_{600} \sim 0.6\right)$ and overexpression of GroEL/ES was induced by the addition of $0.2 \% \mathrm{w} / \mathrm{v}$ arabinose, and further incubation for 2 hours. Cells were resuspended in fresh LB media (without arabinose) with $300 \mathrm{mM} \mathrm{NaCl}$ and 20 $\mathrm{mM}$ L-proline and shaken for 16 hours at $16{ }^{\circ} \mathrm{C}$. Pelleted cells were resuspended in $\mathrm{pH} 8.0$ Lysis Buffer $(20 \mathrm{mM}$ sodium phosphate, $300 \mathrm{mM} \mathrm{NaCl}, 10 \mathrm{mM}$ imidazole, $5 \%$ glycerol, $2 \mathrm{mM}$ $\mathrm{MgCl}_{2}$ ) with $\sim 5 \mathrm{mM}$ PMSF and subject to a freeze-thaw cycle at $-80{ }^{\circ} \mathrm{C}$ before lysis by use of a Microfluidizer high pressure homogenizer. The soluble fraction was collected by centrifugation (15,000 RCF, $20 \mathrm{~min}$ ) and 0.2/0.8 $\mu \mathrm{m}$ filtered. Clarified cell lysate was batch-bound to preequilibrated Ni-NTA resin as described above. Columns were washed thoroughly before resuspension in bdSENP1 Reaction Buffer $(20 \mathrm{mM}$ sodium phosphate $\mathrm{pH} 8.0,300 \mathrm{mM} \mathrm{NaCl}, 1$ mM DTT, 10\% glycerol). Purified bdSENP1 was added to resuspended columns and rocked gently overnight at $4{ }^{\circ} \mathrm{C}$ to facilitate cleavage of the His ${ }_{14}$-bdSUMO tag from the target protein. Flow-through from the bdSENP1 reaction was applied to a $5 \mathrm{~mL}$ HiTrap Q FF column equilibrated in Q Buffer A (100 mM HEPES pH 8.0). Protein was eluted off the column over a linear $\mathrm{NaCl}$ gradient from $5 \mathrm{mM}$ to $1 \mathrm{M}$. Eluted fractions were analyzed by SDS-PAGE prior to concentration and separation by size exclusion chromatography using a Superose 6 Increase 10/300 GL column (GE Healthcare Life Sciences, Marlborough, MA) equilibrated in SEC Buffer (50 mM sodium phosphate $\mathrm{pH} 8.0,300 \mathrm{mM} \mathrm{NaCl}, 25 \mathrm{mM} \mathrm{MgCl} 2,1 \mathrm{mM} \mathrm{DTT}, 5 \mathrm{mM} \mathrm{NaHCO}$ ). Eluted SEC fractions were analyzed by SDS-PAGE and Native PAGE for Rubisco content and purity. Samples were stored in $20 \mathrm{mM}$ sodium phosphate $\mathrm{pH} 8.0,150 \mathrm{mM} \mathrm{NaCl}, 10 \mathrm{mM} \mathrm{MgCl}$, $10 \mathrm{mM} \mathrm{NaHCO}$ at $-80{ }^{\circ} \mathrm{C}$. 
Syn6301 RbcLS was prepared in a similar fashion to previous reports ${ }^{21,40}$. Plasmids Syn6301-rbcLS-pET11A and pBADES/EL were co-transformed into BL21 DE3 Star E. coli cells. Cells were grown to mid-log phase at $30{ }^{\circ} \mathrm{C}\left(\mathrm{OD}_{600} \sim 0.6\right)$ and overexpression of GroEL/ES was induced by $0.4 \% \mathrm{w} / \mathrm{v}$ arabinose for 1.5 hours. Cells were resuspended in fresh media (without arabinose) and induced with $1 \mathrm{mM}$ IPTG for 16 hours at $16{ }^{\circ} \mathrm{C}$. Cells were lysed by using a Microfluidizer high pressure homogenizer and centrifuged (15,000 RCF, 20 minutes). Soluble protein from whole-cell lysate was $0.2 / 0.8 \mu \mathrm{m}$ filtered and subject to ammonium sulfate precipitation at the $30-40 \%$ cut (where the protein is soluble at $30 \% \mathrm{w} / \mathrm{v}$ ammonium sulfate, but precipitates at $40 \%$ saturation. Precipitated protein was resuspended in $\mathrm{pH} 8.0$ Lysis Buffer, desalted, and applied to a MonoQ 10/100 GL column (GE Healthcare Life Sciences, Marlborough, MA) equilibrated in Q Buffer A. Protein was eluted off the column over a linear $\mathrm{NaCl}$ gradient from $5 \mathrm{mM}$ to $1 \mathrm{M}$. Eluted fractions were analyzed by SDS-PAGE prior to concentration and size exclusion chromatography as described for P. breve Rubisco. Samples were stored in $20 \mathrm{mM}$ sodium phosphate $\mathrm{pH} 8.0,150 \mathrm{mM} \mathrm{NaCl}, 10 \mathrm{mM} \mathrm{MgCl}, 10 \mathrm{mM}$ $\mathrm{NaHCO}_{3}$ at $-80{ }^{\circ} \mathrm{C}$.

Syn6301 RbcL expressed without RbcS was prepared in a similar fashion to previous reports $^{21,40}$. Plasmids Syn6301-rbcL-pET11A and pG-KJE8 were co-transformed into BL21 DE3 Star E. coli cells. Cells were grown to mid-log phase at $30{ }^{\circ} \mathrm{C}\left(\mathrm{OD}_{600} \sim 0.6\right)$ and overexpression of dnaK/dnaJ/grpE was induced by $0.4 \% \mathrm{w} / \mathrm{v}$ arabinose for 2 hours. Cells were resuspended in fresh media (without arabinose) and induced with $1 \mathrm{mM}$ IPTG for 16 hours at $16{ }^{\circ} \mathrm{C}$. Cells were lysed and centrifuged as described for Syn6301 RbcLS. Soluble protein from whole-cell lysate was subject to ammonium sulfate precipitation at the 50-60\% cut. Precipitated protein at $60 \%$ saturation was resuspended in lysis buffer and purified via anion exchange and size exclusion chromatography, then stored at $-80{ }^{\circ} \mathrm{C}$ as described for Syn6301 RbcLS.

PAGE analyses. Rubisco samples were activated with excess $\mathrm{NaHCO}_{3}$ and incubated with 10fold molar excess 2-carboxyarabinitol 1,5-bisphosphate (2CABP) as described previously ${ }^{30}$. 2CABP was synthesized according to previously described methods ${ }^{60,61}$. SDS-PAGE samples were prepared according to standard procedures in Laemmli Sample Buffer (Bio-rad, Hercules, CA) with 2-mercaptoethanol, and heated at $98{ }^{\circ} \mathrm{C}$ for 5 minutes, followed by centrifugation in a benchtop centrifuge at maximum speed for 1 minute. Samples were resolved on 12\% MiniPROTEAN® ${ }^{\circledR}$ TGX $^{\mathrm{TM}}$ precast protein gels (Bio-rad) in 1x Tris/Glycine/SDS buffer (Bio-Rad) and stained in AcquaStain (Bulldog Bio, Portsmouth, NH). Non-denaturing PAGE samples were prepared by mixing protein with Native Sample Buffer (Bio-Rad) at $4{ }^{\circ} \mathrm{C}$. Samples were resolved at $4{ }^{\circ} \mathrm{C}$ on $4-15 \%$ Mini PROTEAN ${ }^{\circledR} \mathrm{TGX}^{\mathrm{TM}}$ precast protein gels (Bio-rad) in $1 \mathrm{x}$ Tris/Glycine buffer (Bio-Rad) and visualized by staining with AcquaStain.

Crystallization, X-ray data collection, and structure determination. For crystallography, $\mathrm{P}$. breve Rubisco was prepared as described above, but with a final buffer composition of $100 \mathrm{mM}$ HEPES-OH pH 8.0, $100 \mathrm{mM} \mathrm{NaCl}, 25 \mathrm{mM} \mathrm{MgCl}_{2}, 1 \mathrm{mM}$ DTT, $5 \mathrm{mM} \mathrm{NaHCO}$. Samples at 10- 

were incubated for $1 \mathrm{hr}$ at ambient temperature in the presence of a 10-fold molar excess of $2 \mathrm{CABP}$ before setting up crystal trays. P. breve Rubisco protein was screened using the crystallization screens: Berkeley Screen ${ }^{62}$, Crystal Screen, SaltRx, PEG/Ion, Index and PEGRx (Hampton Research, Aliso Viejo, CA). The crystals of P. breve Rubisco were found in $0.1 \mathrm{M}$ Tris $\mathrm{pH} 8.0$ and $30 \%$ Polyethylene glycol monomethyl ether 2,000 obtained by the sitting-drop vapor-diffusion method with drops consisting of a mixture of $0.2 \mu \mathrm{L}$ of protein solution and 0.2 $\mu \mathrm{L}$ of reservoir solution.

A crystal of P. breve Rubisco was placed in a reservoir solution containing $20 \%(\mathrm{v} / \mathrm{v})$ glycerol, then flash-cooled in liquid nitrogen. The X-ray data sets for P. breve Rubisco were collected at the Berkeley Center for Structural Biology beamline 8.2.2 of the Advanced Light Source at Lawrence Berkeley National Laboratory (LBNL). The diffraction data were recorded using an ADSC-Q315r detector. The data sets were processed using the program Xia2 ${ }^{63}$.

The P. breve Rubisco crystal structure was determined by the molecular-replacement method with the program PHASER ${ }^{64}$ within the Phenix suite ${ }^{65,66}$, using as a search model the structure of a Rubisco from Thermosynechococcus elongatus (PDB code 2YBV), which shows $57 \%$ sequence identity to the target. The atomic positions obtained from molecular replacement and the resulting electron density maps were used to build the P. breve Rubisco structure and initiate crystallographic refinement and model rebuilding. Structure refinement was performed using the phenix.refine program ${ }^{66}$. Translation-libration-screw (TLS) refinement was used, with each protein chain assigned to a separate TLS group. Manual rebuilding using $\mathrm{COOT}^{67}$ and the addition of water molecules allowed construction of the final model. The final model of P. breve Rubisco has an $\mathrm{R}$ factor of $18.8 \%$ and an $\mathrm{R}_{\text {free }}$ of $22.5 \%$. Root-mean-square deviation differences from ideal geometries for bond lengths, angles and dihedrals were calculated with Phenix. The stereochemical quality of the final model of P. breve Rubisco was assessed by the program MOLPROBITY ${ }^{68}$.

Small-angle X-ray-scattering (SAXS) data collection and analysis. Small-angle X-ray scattering (SAXS) coupled with multi-angle light scattering (MALS) in line with size-exclusion chromatography (SEC) experiments were performed with $50 \mu \mathrm{L}$ samples containing $4.6 \mathrm{mg} / \mathrm{mL}$ of P. breve Rubisco incubated with or without 2CABP prepared in $20 \mathrm{mM}$ HEPES-OH ( $\mathrm{pH} 8.0$ ), $300 \mathrm{mM} \mathrm{NaCl}, 10 \mathrm{mM} \mathrm{MgCl}_{2}, 10 \mathrm{mM} \mathrm{NaHCO}_{3}$. SEC-SAXS-MALS data were collected at the ALS beamline 12.3.1 at Lawrence-Berkeley National $\mathrm{Lab}^{69}$. The X-ray wavelength was set at $\lambda=1.127 \AA$ and the sample-to-detector distance was $2100 \mathrm{~mm}$ resulting in scattering vectors (q) ranging from $0.01 \AA^{-1}$ to $0.4 \AA^{-1}$. The scattering vector is defined as $q=4 \pi \sin \theta / \lambda$, where $2 \theta$ is the scattering angle. All experiments were performed at $20{ }^{\circ} \mathrm{C}$ and the data was processed as described $^{70}$. Briefly, a SAXS flow cell was directly coupled with an online 1260 Infinity HPLC system (Agilent, Santa Clara, CA) using a Shodex KW804 column (Showa Denko, Tokyo, 

$\mathrm{NaCl}, 10 \mathrm{mM} \mathrm{MgCl} 2,10 \mathrm{mM} \mathrm{NaHCO} 3$ ) with a flow rate of $0.5 \mathrm{~mL} / \mathrm{min} .90 \mu \mathrm{L}$ of sample was separated by SEC, and three second X-ray exposures were collected continuously during a 30 min elution. The SAXS frames recorded prior to sample analysis were subtracted from all other frames. The subtracted frames were investigated by radius of gyration $(\mathrm{Rg})$ derived by the Guinier approximation, $I(q)=I(0) \exp \left(-q^{2} \operatorname{Rg} 2 / 3\right)$ with the limits $q R g<1$.6. The elution peak was mapped by comparing integral of ratios to background and $\mathrm{Rg}$ relative to the recorded frame using the program SCÅTTER. Uniform $\mathrm{Rg}$ values across an elution peak represent a homogenous assembly. Final merged SAXS profiles, derived by integrating multiple frames across the elution peak, were used for further analysis including Guinier plot which determined aggregation free state. The program SCATTER was used to compute the pair distribution, or $\mathrm{P}(\mathrm{r})$, functions presented in Figure 3B. $\mathrm{P}(\mathrm{r})$ functions were normalized based on the molecular weight determined by SCÅTTER using volume of correlation $\mathrm{Vc}^{49}$ (Supplementary Table 2). Eluent was subsequently split 3:1 between the SAXS line and a series of UV detectors at 280 and $260 \mathrm{~nm}$, a MALS detector, a quasi-elastic light scattering (QELS) detector, and a refractometer detector. MALS experiments were performed using an 18-angle DAWN HELEOS II light scattering detector connected in tandem to an Optilab refractive index concentration detector (Wyatt Technology, Goleta, CA). System normalization and calibration was performed with bovine serum albumin using a $45 \mu \mathrm{L}$ sample at $10 \mathrm{mg} / \mathrm{mL}$ in SEC Buffer and a dn/dc value of 0.19. The light scattering experiments were used to perform analytical scale chromatographic separations for M.W. determination of the principal peaks in the SEC analysis. UV, MALS, and differential refractive index data was analyzed using Wyatt ASTRA 7 software to monitor the homogeneity of the sample across the elution peak complementary to the above-mentioned SECSAXS signal validation.

SAXS modeling. The atomistic model of $\mathrm{P}$. breve Rubisco in the open conformation was prepared based on the crystal structure of the closed conformation presented in this study by including missing $\mathrm{N}$ - and $\mathrm{C}$-terminal residues using the program MODELLER ${ }^{71}$. Different extensions and compactions of the unfolded tails were built to screen conformational variability. The experimental SAXS profiles were then compared to theoretical scattering curves generated from these atomistic models using $\mathrm{FoXS}^{57,58}$. Theoretical scattering profiles were used to calculate $\mathrm{P}(\mathrm{r})$ functions and further compared to experimental $\mathrm{P}(\mathrm{r})$ functions to validate solution state conformations of $\mathrm{P}$. breve Rubisco.

Negative-staining electron microscopy. $3 \mu \mathrm{L}$ of $1 \mathrm{mg} / \mathrm{mL}$ P. breve Rubisco in SEC Buffer were applied to a glow-discharged carbon grid $(30 \mathrm{~mA}, 30 \mathrm{sec})$ and incubated for $1 \mathrm{~min}$ at room temperature. Five drops of $2 \%$ uranyl acetate were then sequentially applied and blotted off for negative staining. 50 images were taken on a JEOL $2100 \mathrm{~F}$ at $\mathrm{x} 40,000$ nominal magnification, $200 \mathrm{kV}$, with $1.48 \AA$ Apixel sampling on a DE-20 detector. 4062 particles were selected and 2-D 497 classified using cisTEM. 
Rubisco activity assays. Rubisco specificity was determined using the method of Parry et al. ${ }^{24}$, with the exception that the activation buffer included $250 \mathrm{mM} \mathrm{NaCl}$ to enhance the solubility of P. breve Form I' Rubisco, and pKa of 6.11 was used for calculations. Measurements using $T$. aestivum (bread wheat) Rubisco were used for normalization as previously described ${ }^{24}$, and results from testing with $T$. aestivum Rubisco showed no effect of $\mathrm{NaCl}$ in the activation buffer.

504 Purified Rubisco was used to determine catalytic properties as described previously ${ }^{72}$, with the

505 following alterations to protein desalting and activation: an aliquot of concentrated Rubisco was diluted with an activation mix containing $100 \mathrm{mM}$ Bicine- $\mathrm{NaOH} \mathrm{pH} 8.0,20 \mathrm{mM} \mathrm{MgCl} 2,250$ $\mathrm{mM} \mathrm{NaCl}, 10 \mathrm{mM} \mathrm{NaHCO}$, and $1 \%$ (v/v) Plant Protease Inhibitor cocktail (Sigma-Aldrich, $\mathrm{UK}$ ). This was then incubated on ice for $20 \mathrm{~min}$ before used to assay at $\mathrm{CO}_{2}$ concentrations of $20,40,60,120,280$, and $400 \mu \mathrm{M}$. These were combined with $\mathrm{O}_{2}$ concentrations of either 0,21 , 40 , or $70 \%(\mathrm{v} / \mathrm{v})$ to determine $K_{O} . V_{O}$ was calculated from measured parameters using the equation $S_{C / O}=\left(V_{C} / K_{C}\right) /\left(V_{O} / K_{O}\right)$. $V_{C}$ was determined using measurements with $0 \% \mathrm{O}_{2}$. An aliquot of the activated protein was used for determination of Rubisco active sites via ${ }^{14} \mathrm{C}-\mathrm{CABP}$ binding using the method of Sharwood et al. ${ }^{73}$ with $250 \mathrm{mM} \mathrm{NaCl}$, instead of the typical $75 \mathrm{mM}$, in the activation buffer.

Protein thermal shift (PTS) assay. The PTS assay was conducted using a Protein Thermal Shift ${ }^{\mathrm{TM}}$ kit (Thermo Fisher, Waltham, MA). Samples were prepared with $1 \mathrm{mg} / \mathrm{mL}$ protein in $1 \mathrm{x}$ PTS phosphate buffer, and 4x PTS dye in Thermo Fisher MicroAmp Optical 8-Tube Strips. Assay was conducted on an Applied Biosciences QuantStudio 3 RT-PCR machine. The assay consisted of initial cooling and hold at $16{ }^{\circ} \mathrm{C}$ for 1 minute, followed by an $0.05^{\circ} \mathrm{C} / \mathrm{s}$ increase to $95{ }^{\circ} \mathrm{C}$, and a final hold at $95{ }^{\circ} \mathrm{C}$ for 1 minute. Data was analyzed in Protein Thermal Shift ${ }^{\mathrm{TM}}$ Software.

Other software. Structure-based sequence alignments were conducted using PROMALS3D ${ }^{53}$ and $\mathrm{MAFFT}^{54}$. Analyses of protein amino acid contacts and subunit interface thermodynamics were performed using CCP4 CONTACTS ${ }^{55}$, and PISA ${ }^{74,75}$, respectively. UCSF Chimera ${ }^{76}$ was utilized for the visualization of protein models, generating electrostatic potential maps, and the 529 preparation of manuscript figures. 
530 Data availability. Form I' RbcL amino acid sequences are included as a supplementary file 531 (Supplementary data 1). Sequences used to generate Fig. 1a were uploaded to figshare (DOI: $53210.6084 / \mathrm{m} 9$.figshare.9980630) along with the associated phylogenetic tree. Representative MAG genbank scaffolds are included as a supplementary file (Supplementary data 2). Site-directed mutagenesis primers and synthesized candidate Form I' $r b c L$ genes are included as a supplementary file (Supplementary data 3). The structural coordinates of 2CABP-bound P. breve Rubisco have been deposited in the PDB under the accession ID 6URA. The crystal structure of Syn6301 Rubisco can be found on the PDB under the accession ID 1RBL. Publicly available databases used in this study include: PDB (www.rcsb.org), pfam (www.pfam.xfam.org), TIGRfams (www.tigrfams.jcvi.org), and KEGG database (www.genome.jp/kegg.html).Two Chloroflexi genomes identified in this study are available at:

https://ggkbase.berkeley.edu/Chloroflexi_Rubisco_PatrickShih/organisms.

Materials \& correspondence. Correspondence and material requests should be addressed to P.M. Shih and J.F. Banfield

\section{Author information}

Author contributions. D.M.B, A.K.L., and P.M.S. designed experiments. D.M.B and A.K.L. prepared all protein samples, performed all PAGE analyses, and protein thermal shift experiments. M.H. performed all SEC-SAXS-MALS experiments and data analysis. J.H.P. performed X-ray crystallography data acquisition, image processing, and structure determination. D.M.B. performed all structural analyses. A.K.L. performed all site-directed mutagenesis experiments. D.J.O. performed all Rubisco activity and kinetic measurements. C. H. and J.F.B performed all metagenomic and phylogenetic analyses. All authors participated in writing and manuscript preparation.

Acknowledgments. D.M.B, A.K.L., and P.M.S. acknowledge support from a Society in Science-Branco Weiss fellowship from ETH Zurich. J.H.P., P.D.A., and P.M.S. acknowledge support from the Joint BioEnergy Institute which is supported by the US Department of Energy, Office of Science, Office of Biological and Environmental Research under Contract No. DEAC02-05CH11231 between Lawrence Berkeley National Laboratory and the US Department of Energy. C.H. and J.F.B. thank Adi Lavy and Allison Sharrar for providing unpublished Rubisco sequences, Jacob West-Roberts for assistance, the Rifle IFRC/SFA 2.0 Metagenomics and Proteomics Data Analysis Project, the Allen Foundation, the Chan Zuckerberg Biohub, and the Innovative Genomics Institute for support. C.H. acknowledges the Camille and Henry Dreyfus Foundation for a postdoctoral fellowship, and the Joint Genome Institute CSP for sequencing. M.H. acknowledge support from the Department of Energy BER Integrated Diffraction Analysis Technologies (IDAT) program, NIGMS grant P30 GM124169-01, and ALS-ENABLE for SAXS 
570 data collection at SIBYLS. D.J.O., M.A.J.P., and E.C.S. acknowledge support from the UK 571 Biotechnology and Biological Sciences Research Council (BBSRC; grant number $572 \mathrm{BB} / \mathrm{I024488/1)}$. We would like to thank Manajit Hayer-Hartl (Max Planck Institute of 573 Biochemistry, Martinsried, Germany) for the kind donation of the Syn6301-rbcL-pET11a, 574 Syn6301-rbcLS-pET11a, pG-KJE8, and pBAD33ES/EL plasmids used in this study. 575 Additionally, we would like to thank Noam Prywes for the kind donation of the pET28-His 14- $^{-}$ 576 bdSUMO and pSF1389 plasmids. We would also like to thank Fei Guo and the UC Davis

577 BioEM core facility for EM images, and the laboratory of Justin Siegel (UC Davis Genome 578 Center) for use of their qPCR machine for protein thermal shift experiments. We are grateful to 579 Avi Flamholz for collecting publicly available Form I Rubisco kinetic data used in this study, 580 and to Alyssa Marinas and Rick Vermon Callado for assisting with enzyme purifications. We 581 thank Kasey Markel for his edits and suggestions on the manuscript.

582

585 The authors declare no competing interests.

586

587

588

2. Tabita, F. R. et al. Function, Structure, and Evolution of the RubisCO-Like Proteins and

\section{References}

1. Nisbet, E. G. et al. The age of Rubisco: the evolution of oxygenic photosynthesis.

\section{Declarations of interest} Geobiology 5, 311-335 (2007). Their RubisCO Homologs. Microbiol. Mol. Biol. Rev. 71, 576-599 (2007).

3. Tabita, F. R., Satagopan, S., Hanson, T. E., Kreel, N. E. \& Scott, S. S. Distinct form I, II, III, and IV Rubisco proteins from the three kingdoms of life provide clues about Rubisco evolution and structure/function relationships. J. Exp. Bot. 59, 1515-1524 (2007).

4. Andrews, T. J. Catalysis by cyanobacterial ribulose-bisphosphate carboxylase large subunits in the complete absence of small subunits. J. Biol. Chem. 263, 12213-12219 (1988).

5. Morell, M. K., Wilkin, J. M., Kane, H. J. \& Andrews, T. J. Side reactions catalyzed by ribulose-bisphosphate carboxylase in the presence and absence of small subunits. J. Biol. Chem. 272, 5445-5451 (1997).

600 6. Spreitzer, R. J. Role of the small subunit in ribulose-1,5-bisphosphate 
carboxylase/oxygenase. Archives of Biochemistry and Biophysics vol. 414 141-149 (2003).

602 7. Joshi, J., Mueller-Cajar, O., Tsai, Y.-C. C., Hartl, F. U. \& Hayer-Hartl, M. Role of Small 603 Subunit in Mediating Assembly of Red-type Form I Rubisco. J. Biol. Chem. 290, 1066$604 \quad 1074(2015)$

605 8. Liu, C. et al. Coupled chaperone action in folding and assembly of hexadecameric Rubisco. $606 \quad$ Nature 463, 197-202 (2010).

607 9. Grabsztunowicz, M., Górski, Z., Luciński, R. \& Jackowski, G. A reversible decrease in 608 ribulose 1,5-bisphosphate carboxylase/oxygenase carboxylation activity caused by the 609 aggregation of the enzyme's large subunit is triggered in response to the exposure of 610 moderate irradiance-grown plants to low irradiance. Physiol. Plant. 154, 591-608 (2015).

611 10. Kusian, B. \& Bowien, B. Organization and regulation of cbb CO2 assimilation genes in 612 autotrophic bacteria. FEMS Microbiol. Rev. 21, 135-155 (1997).

613 11. Tabita, F. R. Microbial ribulose 1,5-bisphosphate carboxylase/oxygenase: A different $614 \quad$ perspective. Photosynth. Res. 60, 1-28 (1999).

615 12. Whitney, S. M. \& Andrews, T. J. The gene for the ribulose-1,5-bisphosphate 616 carboxylase/oxygenase (Rubisco) small subunit relocated to the plastid genome of tobacco 617 directs the synthesis of small subunits that assemble into Rubisco. Plant Cell 13, 193-205 $618 \quad$ (2001).

619 13. Bryant, D. A. \& Liu, Z. Chapter Four - Green Bacteria: Insights into Green Bacterial 620 Evolution through Genomic Analyses. in Advances in Botanical Research (ed. Beatty, J. T.) 621 vol. 66 99-150 (Academic Press, 2013).

622 14. Shih, P. M., Ward, L. M. \& Fischer, W. W. Evolution of the 3-hydroxypropionate bicycle 623 and recent transfer of anoxygenic photosynthesis into the Chloroflexi. Proc. Natl. Acad. Sci. 
U. S. A. 114, 10749-10754 (2017).

625 15. Ward, L. M., Hemp, J., Shih, P. M., McGlynn, S. E. \& Fischer, W. W. Evolution of

626 Phototrophy in the Chloroflexi Phylum Driven by Horizontal Gene Transfer. (2018)

627 doi:10.3389/fmicb.2018.00260.

628 16. Fischer, W. W., Hemp, J. \& Johnson, J. E. Evolution of Oxygenic Photosynthesis. Annu.

629 Rev. Earth Planet. Sci. 44, 647-683 (2016).

630 17. Roy, H. Rubisco assembly: a model system for studying the mechanism of chaperonin $631 \quad$ action. Plant Cell 1, 1035-1042 (1989).

632 18. Hayer-Hartl, M. From chaperonins to Rubisco assembly and metabolic repair. Protein Sci. $633 \quad 26,2324-2333(2017)$.

634 19. Aigner, H. et al. Plant RuBisCo assembly in E. coli with five chloroplast chaperones including BSD2. Science 358, 1272-1278 (2017).

636 20. Wilson, R. H. \& Hayer-Hartl, M. Complex Chaperone Dependence of Rubisco Biogenesis. Biochemistry 57, 3210-3216 (2018).

638 21. Saschenbrecker, S. et al. Structure and Function of RbcX, an Assembly Chaperone for 639 Hexadecameric Rubisco. Cell 129, 1189-1200 (06/2007).

640 22. Gunn, L. H., Valegård, K. \& Andersson, I. A unique structural domain in 641 Methanococcoides burtonii ribulose-1,5-bisphosphate carboxylase/oxygenase (Rubisco) 642 acts as a small subunit mimic. J. Biol. Chem. 292, 6838-6850 (2017).

643 23. Goloubinoff, P., Christeller, J. T., Gatenby, A. A. \& Lorimer, G. H. Reconstitution of active 644 dimeric ribulose bisphosphate carboxylase from an unfolded state depends on two 645 chaperonin proteins and Mg-ATP. Nature 342, 884-889 (1989).

646 24. Parry, M. A. J., Keys, A. J. \& Gutteridge, S. Variation in the Specificity Factor of C3 
Higher Plant Rubiscos Determined by the Total Consumption of Ribulose-P2. J. Exp. Bot. 40, 317-320 (1989).

25. Tcherkez, G. G. B., Farquhar, G. D. \& Andrews, T. J. Despite slow catalysis and confused substrate specificity, all ribulose bisphosphate carboxylases may be nearly perfectly optimized. Proceedings of the National Academy of Sciences 103, 7246-7251 (2006).

652 26. Flamholz, A. I. et al. Revisiting Trade-offs between Rubisco Kinetic Parameters. Biochemistry 58, 3365-3376 (2019).

654 27. Yamada, T. \& Sekiguchi, Y. Cultivation of uncultured chloroflexi subphyla: significance 655 and ecophysiology of formerly uncultured chloroflexi 'subphylum i' with natural and biotechnological relevance. Microbes Environ. 24, 205-216 (2009).

657 28. Hemp, J., Ward, L. M., Pace, L. A. \& Fischer, W. W. Draft Genome Sequence of Ornatilinea apprima P3M-1, an Anaerobic Member of the Chloroflexi Class Anaerolineae. Genome Announc. 3, (2015).

29. Ward, L. M., Hemp, J., Pace, L. A. \& Fischer, W. W. Draft Genome Sequence of Leptolinea tardivitalis YMTK-2, a Mesophilic Anaerobe from the Chloroflexi Class Anaerolineae. Genome Announc. 3, (2015).

30. Alonso, H., Blayney, M. J., Beck, J. L. \& Whitney, S. M. Substrate-induced assembly of 664 Methanococcoides burtonii D-ribulose-1,5-bisphosphate carboxylase/oxygenase dimers into decamers. J. Biol. Chem. 284, 33876-33882 (2009).

31. Knott, G. J. et al. Structural basis for AcrVA4 inhibition of specific CRISPR-Cas12a. Elife 8, (2019).

32. Duff, A. P., Andrews, T. J. \& Curmi, P. M. The transition between the open and closed states of rubisco is triggered by the inter-phosphate distance of the bound bisphosphate. $J$. 
Mol. Biol. 298, 903-916 (2000).

671 33. Newman, J., Branden, C. I. \& Jones, T. A. Structure determination and refinement of 672 ribulose 1,5-bisphosphate carboxylase/oxygenase from Synechococcus PCC6301. Acta $673 \quad$ Crystallogr. D Biol. Crystallogr. 49, 548-560 (1993).

674 34. Lu, Z., Zhao, Z. \& Fu, B. Efficient protein alignment algorithm for protein search. BMC 675 Bioinformatics 11 Suppl 1, S34 (2010).

676 35. Cleland, W. W., Andrews, T. J., Gutteridge, S., Hartman, F. C. \& Lorimer, G. H.

677 Mechanism of Rubisco: The Carbamate as General Base ${ }^{x}$. Chem. Rev. 98, 549-562 (1998).

678 36. Andersson, I. \& Backlund, A. Structure and function of Rubisco. Plant Physiol. Biochem. $679 \quad 46,275-291(2008)$.

680 37. van Lun, M., van der Spoel, D. \& Andersson, I. Subunit interface dynamics in 681 hexadecameric rubisco. J. Mol. Biol. 411, 1083-1098 (2011).

682 38. Schneider, G. et al. Comparison of the crystal structures of L2 and L8S8 Rubisco suggests a 683 functional role for the small subunit. EMBO J. 9, 2045-2050 (1990).

684 39. Huynh, K. \& Partch, C. L. Analysis of protein stability and ligand interactions by thermal 685 shift assay. Curr. Protoc. Protein Sci. 79, 28.9.1-14 (2015).

686 40. Greene, D. N., Whitney, S. M. \& Matsumura, I. Artificially evolved Synechococcus 687 PCC6301 Rubisco variants exhibit improvements in folding and catalytic efficiency. Biochem. J 404, 517-524 (2007).

689 41. DePristo, M. A., Weinreich, D. M. \& Hartl, D. L. Missense meanderings in sequence space:

690 a biophysical view of protein evolution. Nat. Rev. Genet. 6, 678-687 (2005).

691 42. Tokuriki, N., Stricher, F., Serrano, L. \& Tawfik, D. S. How protein stability and new

692 functions trade off. PLoS Comput. Biol. 4, e1000002 (2008). 
693 43. Tokuriki, N. \& Tawfik, D. S. Protein dynamism and evolvability. Science 324, 203-207 $694 \quad$ (2009).

695 44. Erb, T. J. \& Zarzycki, J. A short history of RubisCO: the rise and fall (?) of Nature's 696 predominant CO2 fixing enzyme. Curr. Opin. Biotechnol. 49, 100-107 (02/2018).

697 45. Badger, M. R., Hanson, D. \& Dean Price, G. Evolution and diversity of CO2 concentrating 698 mechanisms in cyanobacteria. Funct. Plant Biol. 29, 161-173 (2002).

699 46. Studer, R. A., Christin, P.-A., Williams, M. A. \& Orengo, C. A. Stability-activity tradeoffs 700 constrain the adaptive evolution of RubisCO. Proceedings of the National Academy of $701 \quad$ Sciences 111, 2223-2228 (2014).

702 47. Zhou, Y. \& Whitney, S. Directed Evolution of an Improved Rubisco; In Vitro Analyses to 703 Decipher Fact from Fiction. Int. J. Mol. Sci. 20, (2019).

704 48. Wilson, R. H., Alonso, H. \& Whitney, S. M. Evolving Methanococcoides burtonii archaeal 705 Rubisco for improved photosynthesis and plant growth. Sci. Rep. 6, 22284 (2016).

706 49. Rambo, R. P. \& Tainer, J. A. Accurate assessment of mass, models and resolution by small707 angle scattering. Nature 496, 477-481 (2013).

708 50. Diamond, S. et al. Mediterranean grassland soil C-N compound turnover is dependent on 709 rainfall and depth, and is mediated by genomically divergent microorganisms. Nat $710 \quad$ Microbiol 4, 1356-1367 (2019).

711 51. Lavy, A. et al. Microbial communities across a hillslope-riparian transect shaped by 712 proximity to the stream, groundwater table, and weathered bedrock. Ecol. Evol. 9, 6869$713 \quad 6900(2019)$.

714 52. Knight, S., Andersson, I. \& Brändén, C. I. Crystallographic analysis of ribulose 1,5715 bisphosphate carboxylase from spinach at 2.4 A resolution. Subunit interactions and active 
site. J. Mol. Biol. 215, 113-160 (1990).

717 53. Pei, J., Kim, B.-H. \& Grishin, N. V. PROMALS3D: a tool for multiple protein sequence

718 and structure alignments. Nucleic Acids Res. 36, 2295-2300 (2008).

719 54. Katoh, K., Rozewicki, J. \& Yamada, K. D. MAFFT online service: multiple sequence

720 alignment, interactive sequence choice and visualization. Brief. Bioinform. (2017)

721 doi:10.1093/bib/bbx108.

722 55. Potterton, E., Briggs, P., Turkenburg, M. \& Dodson, E. A graphical user interface to the 723 CCP4 program suite. Acta Crystallogr. D Biol. Crystallogr. 59, 1131-1137 (2003).

724 56. Mueller-Cajar, O., Morell, M. \& Whitney, S. M. Directed Evolution of Rubisco in 725 Escherichia coli Reveals a Specificity-Determining Hydrogen Bond in the Form II Enzyme.

$726 \quad$ Biochemistry 46, 14067-14074 (2007).

727 57. Schneidman-Duhovny, D., Hammel, M. \& Sali, A. FoXS: a web server for rapid 728 computation and fitting of SAXS profiles. Nucleic Acids Res. 38, W540-4 (2010).

729 58. Schneidman-Duhovny, D., Hammel, M., Tainer, J. A. \& Sali, A. Accurate SAXS profile computation and its assessment by contrast variation experiments. Biophys. J. 105, 962-974

731 (2013).

732 59. Frey, S. \& Görlich, D. A new set of highly efficient, tag-cleaving proteases for purifying 733 recombinant proteins. J. Chromatogr. A 1337, 95-105 (2014).

734 60. Kane, H. J., Wilkin, J. M., Portis, A. R. \& John Andrews T. Potent inhibition of ribulose735 bisphosphate carboxylase by an oxidized impurity in ribulose-1,5-bisphosphate. Plant $736 \quad$ Physiol. 117, 1059-1069 (1998).

737 61. Pierce, J., Tolbert, N. E. \& Barker, R. Interaction of ribulosebisphosphate 738 carboxylase/oxygenase with transition-state analogues. Biochemistry 19, 934-942 (1980). 
62. Pereira, J. H., McAndrew, R. P., Tomaleri, G. P. \& Adams, P. D. Berkeley Screen: a set of 96 solutions for general macromolecular crystallization. J. Appl. Crystallogr. 50, 13521358 (2017).

63. Winter, G., Lobley, C. M. C. \& Prince, S. M. Decision making in xia2. Acta Crystallogr. D Biol. Crystallogr. 69, 1260-1273 (2013).

64. McCoy, A. J. et al. Phaser crystallographic software. J. Appl. Crystallogr. 40, 658-674

745 (2007).

65. Adams, P. D. et al. PHENIX: a comprehensive Python-based system for macromolecular structure solution. Acta Crystallogr. D Biol. Crystallogr. 66, 213-221 (2010).

66. Afonine, P. V. et al. Towards automated crystallographic structure refinement with phenix.refine. Acta Crystallogr. D Biol. Crystallogr. 68, 352-367 (2012).

67. Emsley, P. \& Cowtan, K. Coot: model-building tools for molecular graphics. Acta Crystallogr. D Biol. Crystallogr. 60, 2126-2132 (2004).

68. Davis, I. W. et al. MolProbity: all-atom contacts and structure validation for proteins and nucleic acids. Nucleic Acids Res. 35, W375-83 (2007).

69. Dyer, K. N. et al. High-throughput SAXS for the characterization of biomolecules in solution: a practical approach. Methods Mol. Biol. 1091, 245-258 (2014).

70. Hura, G. L. et al. Robust, high-throughput solution structural analyses by small angle X-ray scattering (SAXS). Nat. Methods 6, 606-612 (2009).

71. Sali, A. \& Blundell, T. L. Comparative protein modelling by satisfaction of spatial restraints. J. Mol. Biol. 234, 779-815 (1993). for improving wheat photosynthesis. J. Exp. Bot. 67, 1827-1838 (03/2016). 
762

763

764

765

766

767

768

769

770

771

772

773

774

775

776

777

778

779

780

781

782

783

784

785

786

787

788

789

790

1

7

73. Sharwood, R. E., Ghannoum, O. \& Whitney, S. M. Prospects for improving CO2 fixation in C3-crops through understanding C4-Rubisco biogenesis and catalytic diversity. Curr. Opin. Plant Biol. 31, 135-142 (2016).

74. Krissinel, E. \& Henrick, K. Inference of macromolecular assemblies from crystalline state. J. Mol. Biol. 372, 774-797 (2007).

75. Krissinel, E. Crystal contacts as nature's docking solutions. J. Comput. Chem. 31, 133-143 (2010).

76. Pettersen, E. F. et al. UCSF Chimera--a visualization system for exploratory research and analysis. J. Comput. Chem. 25, 1605-1612 (2004).

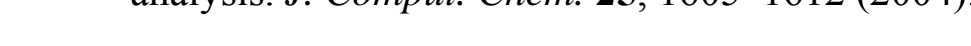

3

4

5

\section{Figure Legends}

Fig. 1. Metagenomics-enabled identification of a novel clade of Form I Rubisco that lack small subunits. a, Maximum likelihood phylogeny of Rubisco RbcL. By including recently discovered metagenome-assembled genomes (MAGs) from Chloroflexi, the emergence of a bona fide, well-supported clade of Rubisco was identified (Form I'). Black circles indicate bootstrap values of 100 and white circles indicate bootstrap values $>90$. b, Example Chloroflexi operons with Form I' Rubisco (dark blue) reveal no presence of a $r b c S$, a defining feature of Form I Rubisco, which are almost always found immediately neighboring $r b c L$ in bacteria; however, other CBB cycle-related genes are found in the operon (light blue). White, other enzymes; gray, hypothetical protein. Annotated loci (i-v) represent Scaffolds 211530, 92, 509483, 467972, and 172446, respectively. For the full annotation information see Supplementary Data 2. GAPDH, glyceraldehyde-3-phosphate dehydrogenase; cbbT, 
transketolase; PRK, phosphoribulokinase; FBP, fructose bisphosphate; TBP, tagatose

$\mathrm{cbbF}$,

fructose

1,6-bisphosphatase.

Fig. 2. Comparison of P. breve kinetic data to reported values of Form I Rubisco. Scatter plots of reported Form I Rubisco kinetic data (black circles) collected at $25^{\circ} \mathrm{C}^{26}$ against P. breve Form I' Rubisco (green dots), including maximum rates of carboxylation and oxygenation of RuBP ( $V_{C}$ and $V_{O}$, respectively), the catalytic efficiency of carboxylation over oxygenation $\left(S_{C / O}\right)$, and Michaelis constants for carboxylation and oxygenation of RuBP $\left(K_{C}\right.$ and $K_{O}$, respectively). Gray dotted lines represent the median for collected Form I Rubisco kinetic data.

Fig. 3. Solution-state characterization of Form I' oligomerization reveals an octameric holoenzyme reminiscent of canonical Form I Rubisco. a, SEC-SAXS-MALS chromatograms of the separation of activated P. breve Rubisco in the absence (top) or presence (bottom) of bound 2CABP. Solid gray lines represent the UV absorbance reading at $280 \mathrm{~nm}$, dashed black lines represent the integrated SAXS signal, while circles represent molecular mass (light blue) data collected from MALS, and Rg values for each SAXS frame (dark blue) versus elution time. b. Experimental P(r) functions determined from SAXS profiles (black dashes) of P. breve Rubisco in the open conformation (light blue) or bound to 2CABP (dark blue). The area under the $\mathrm{P}(\mathrm{r})$ function is normalized relative to the molecular weight estimated by $\mathrm{SAXS}^{49}$ and is listed in Supplementary Table 2. Theoretical P(r) functions are calculated from the theoretical SAXS curves of the corresponding models shown in panel C. The radius where $\mathrm{P}(\mathrm{r})$ approaches zero intensity identifies the maximal dimension of the macromolecule (dashed arrows). c, Surface representation models of P. breve Rubisco with extended (open conformation) or compact (closed conformation) C-terminal regions. d, A representative non-denaturing PAGE gel demonstrating the migration of P. breve Rubisco in the absence (-) or presence $(+)$ of 2CABP. $\mathrm{M}=$ molecular weight marker. Native gel electrophoresis experiment was performed at

Fig. 4. Crystal structure of Form I' Rubisco compared to cyanobacterial Form I Rubisco. Comparison of the structural models of a, Form I Rubisco from Synechococcus sp. strain PCC 6301 (PDB ID: 1RBL) RbcL (green) with RbcS (tan), and b, Form I' Rubisco from P. breve (PDB ID: 6URA, blue) which lacks RbcS. Coulombic electrostatic potential maps of 1RBL ( $\mathrm{RbcS}$ removed) and P. breve Rubisco are illustrated by the charge distributions (negative, red; activity spurred the evolution of the small subunit. a, Salt bridge and hydrogen bond networks present at the dimer-dimer interface of P. breve Rubisco mediate holoenzyme stability in the absence of small subunits. Separate RbcL dimers at the dimer-dimer pair are distinguished by 
831 two separate shades of blue. b, Protein thermal shift assay data with annotated melting 832 temperatures $(\mathrm{Tm})$ for the disassembly of $\mathrm{RbcL}$ dimer quaternary structure from wild-type 833 Syn6301 RbcL, Syn6301 RbcLS, and P. breve RbcL. Reported Tm values represent the average 834 measured from a total of four experiments.

835 
Table 1 | Kinetic characterization of Form I' Rubisco at $25^{\circ} \mathrm{C}$.

\begin{tabular}{|l|l|l|l|l|l|}
\hline Rubisco & $\boldsymbol{V}_{C}\left(\mathbf{s}^{-\mathbf{1}}\right)$ & $\boldsymbol{K}_{\boldsymbol{C}}(\boldsymbol{\mu M})$ & $\boldsymbol{S}_{C / \boldsymbol{O}}$ & $\boldsymbol{V}_{\boldsymbol{O}}\left(\mathbf{s}^{-\mathbf{1}}\right)$ & $\boldsymbol{K}_{\boldsymbol{O}}(\boldsymbol{\mu M})$ \\
\hline Form I' P. breve & $\begin{array}{l}2.23 \pm \\
0.04(5)\end{array}$ & $\begin{array}{l}22.2 \pm \\
9.7(5)\end{array}$ & $\begin{array}{l}36.1 \pm \\
0.9(10)\end{array}$ & $1.11(5)$ & $\begin{array}{l}401 \pm \\
115(5)\end{array}$ \\
\hline $\begin{array}{l}\text { Form I Synechococcus sp. strain } \\
\text { PCC 6301 }\end{array}$ & $\begin{array}{l}14.3 \pm \\
0.71(4)\end{array}$ & $\begin{array}{l}235 \pm \\
20.0(4)\end{array}$ & $\begin{array}{l}56.1 \pm \\
1.3(4)\end{array}$ & $1.10(4)$ & $\begin{array}{l}983 \pm 81 \\
(4)\end{array}$ \\
\hline
\end{tabular}

$V_{C}$ and $V_{O}$ correspond to the maximal rates of the carboxylation and oxygenation reactions, respectively, under saturating substrate concentrations. $K_{C}$ and $K_{O}$ are the Michaelis constants $\left(K_{M}\right)$ for the carboxylation and oxygenation reactions, respectively. $S_{C / O}=\left(V_{C} / K_{C}\right) /\left(V_{O} / K_{O}\right)$. Values represent the mean \pm S.E. with $n$ indicated in parentheses, where $n$ reflects the number of experiments conducted with the same protein sample. 

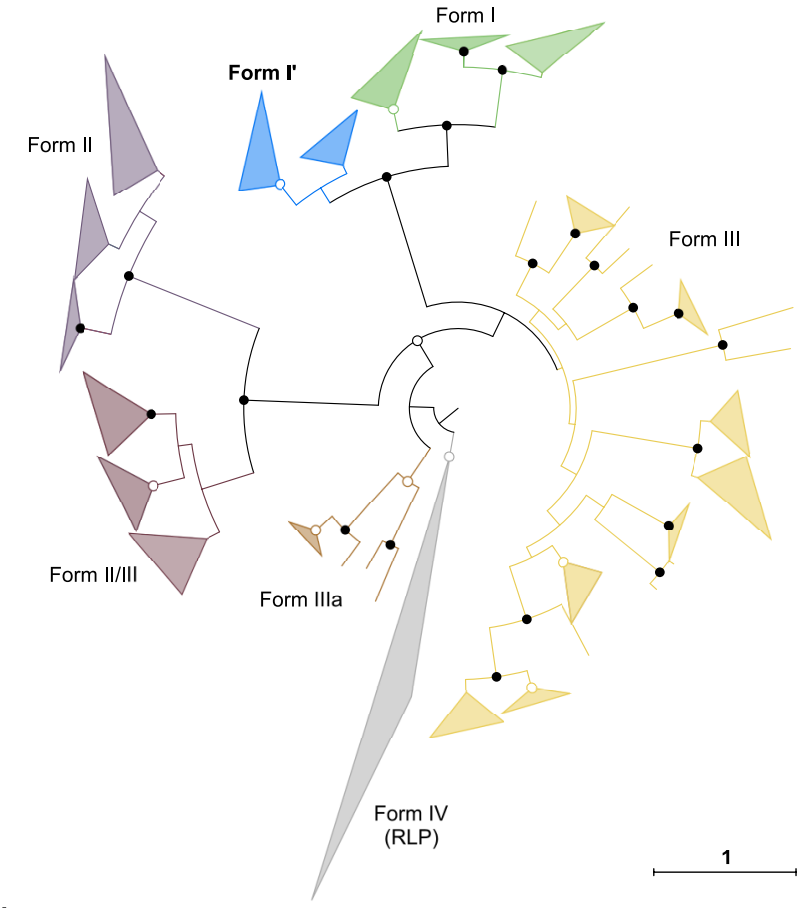

b

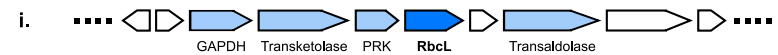

ii.
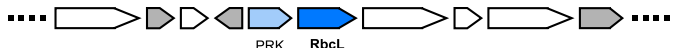

iii.
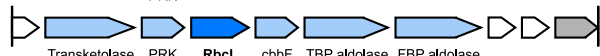

cbbF TBP aldolase FBP aldolase

iv.
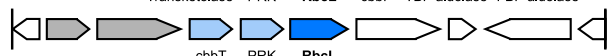

v.

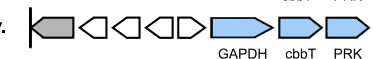

RbcL 
$V_{c}\left(\mathrm{~s}^{-1}\right)$

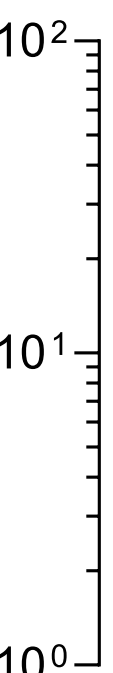

$K_{c}(\mu \mathrm{M})$

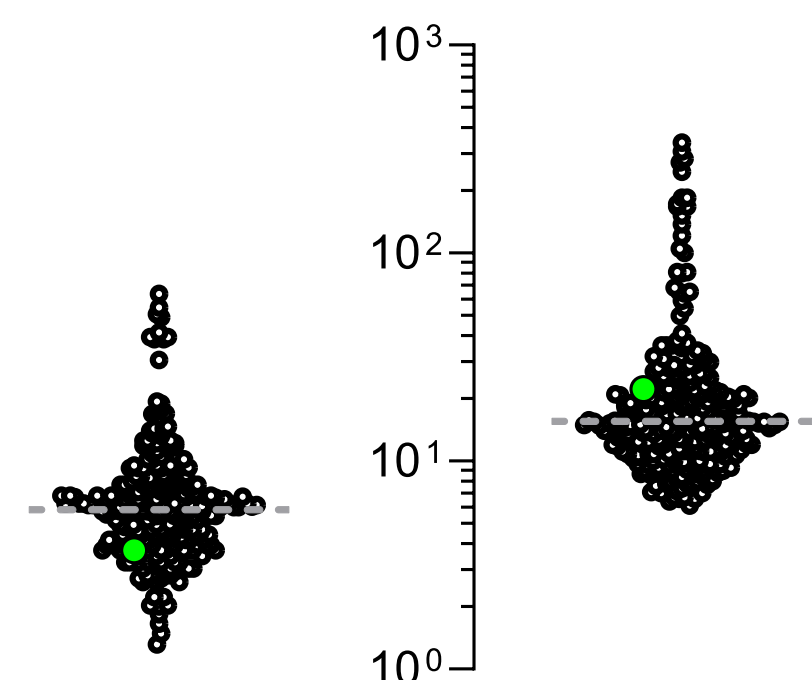

$S_{C / O}$

$V_{o}\left(\mathbf{s}^{-1}\right)$

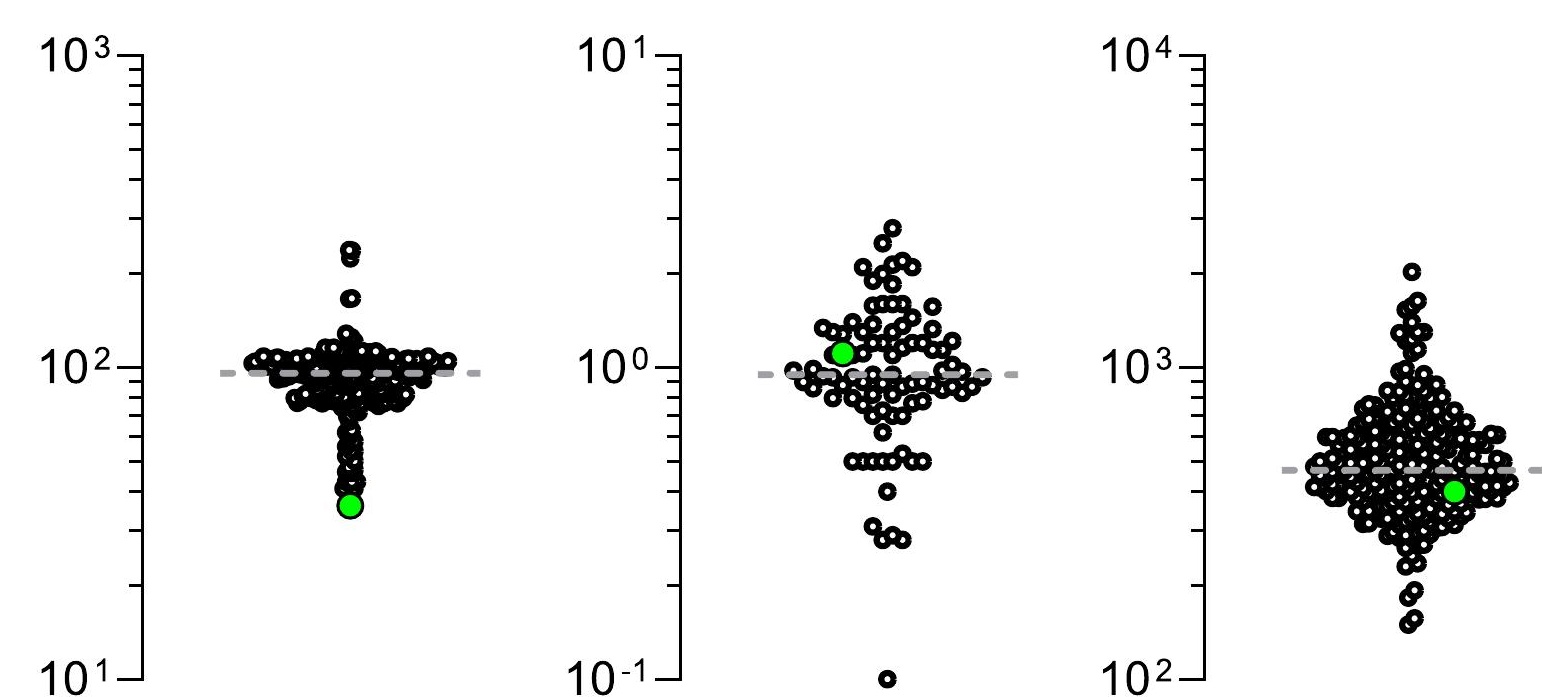

$K_{o}(\mu \mathrm{M})$ 


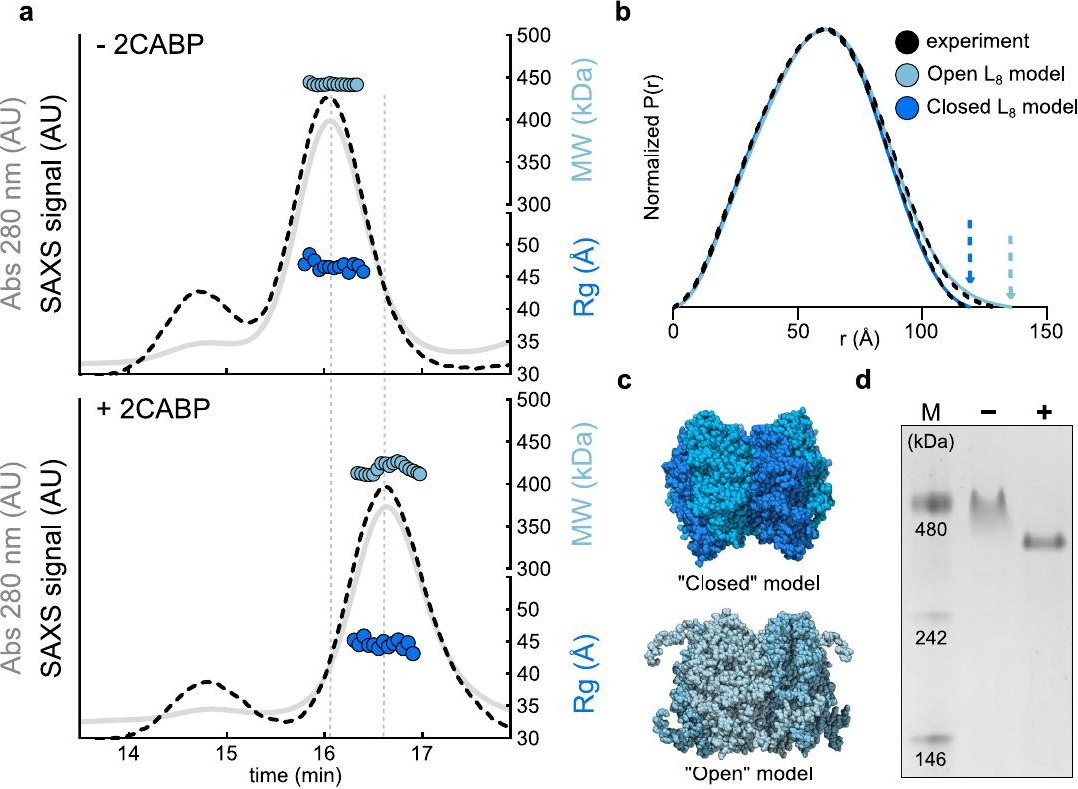




\section{Syn6301 RbcLS ( $\left.\mathrm{L}_{8} \mathrm{~S}_{8}\right)$}

P. breve RbcL (L8)
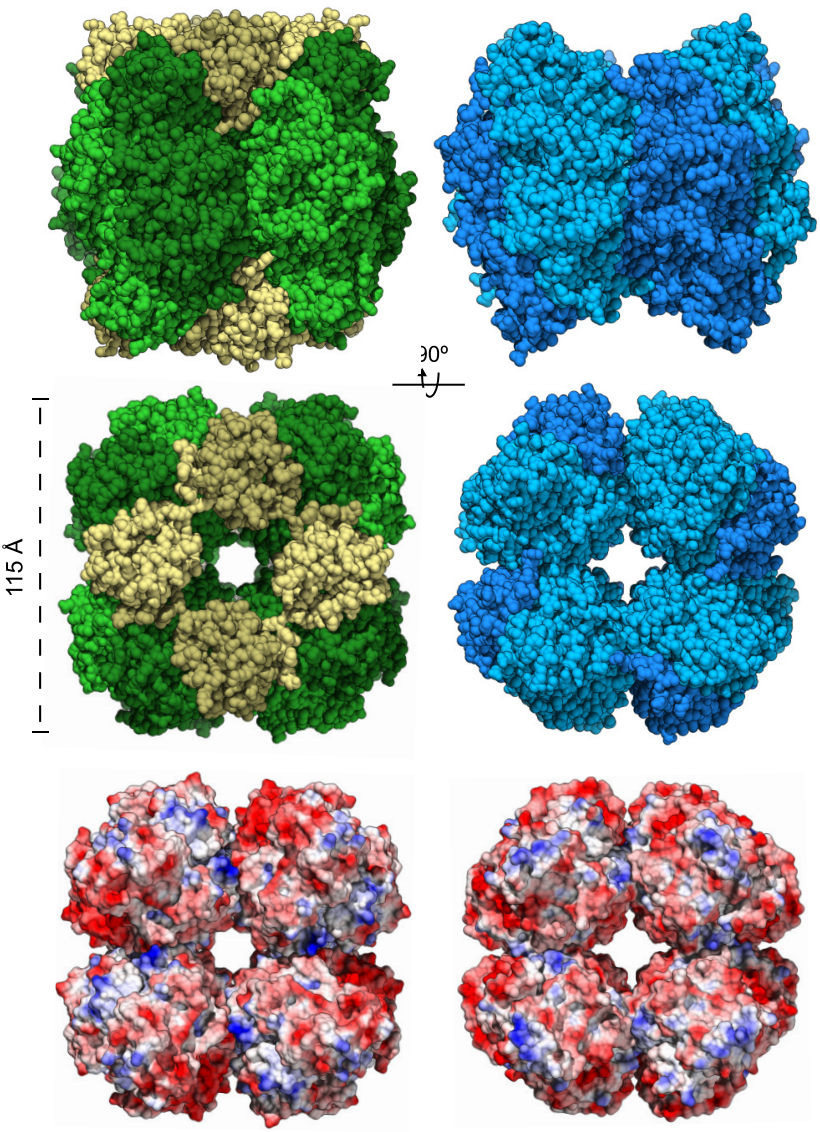



\section{Table 1 | Kinetic characterization of Form I' Rubisco at $25^{\circ} \mathrm{C}$.}

\begin{tabular}{|l|l|l|l|l|l|}
\hline Rubisco & $\boldsymbol{V}_{C}\left(\mathbf{s}^{-1}\right)$ & $\boldsymbol{K}_{C}(\boldsymbol{\mu M})$ & $\boldsymbol{S}_{C / O}$ & $\boldsymbol{V}_{\boldsymbol{O}}\left(\mathbf{s}^{-1}\right)$ & $\boldsymbol{K}_{\boldsymbol{O}}(\boldsymbol{\mu M})$ \\
\hline Form I' P. breve & $\begin{array}{l}2.23 \pm \\
0.04(5)\end{array}$ & $\begin{array}{l}22.2 \pm \\
9.7(5)\end{array}$ & $\begin{array}{l}36.1 \pm \\
0.9(10)\end{array}$ & $1.11(5)$ & $\begin{array}{l}401 \pm \\
115(5)\end{array}$ \\
\hline $\begin{array}{l}\text { Form I Synechococcus sp. strain } \\
\text { PCC 6301 }\end{array}$ & $\begin{array}{l}14.3 \pm \\
0.71(4)\end{array}$ & $\begin{array}{l}235 \pm \\
20.0(4)\end{array}$ & $\begin{array}{l}56.1 \pm \\
1.3(4)\end{array}$ & $1.10(4)$ & $\begin{array}{l}983 \pm 81 \\
(4)\end{array}$ \\
\hline
\end{tabular}

$V_{C}$ and $V_{O}$ correspond to the maximal rates of the carboxylation and oxygenation reactions, respectively, under saturating substrate concentrations. $K_{C}$ and $K_{O}$ are the Michaelis constants $\left(K_{M}\right)$ for the carboxylation and oxygenation reactions, respectively. $S_{C / O}=\left(V_{C} / K_{C}\right) /\left(V_{O} / K_{O}\right)$. Values represent the mean \pm S.E. with $n$ indicated in parentheses, where $n$ reflects the number of experiments conducted with the same protein sample. 


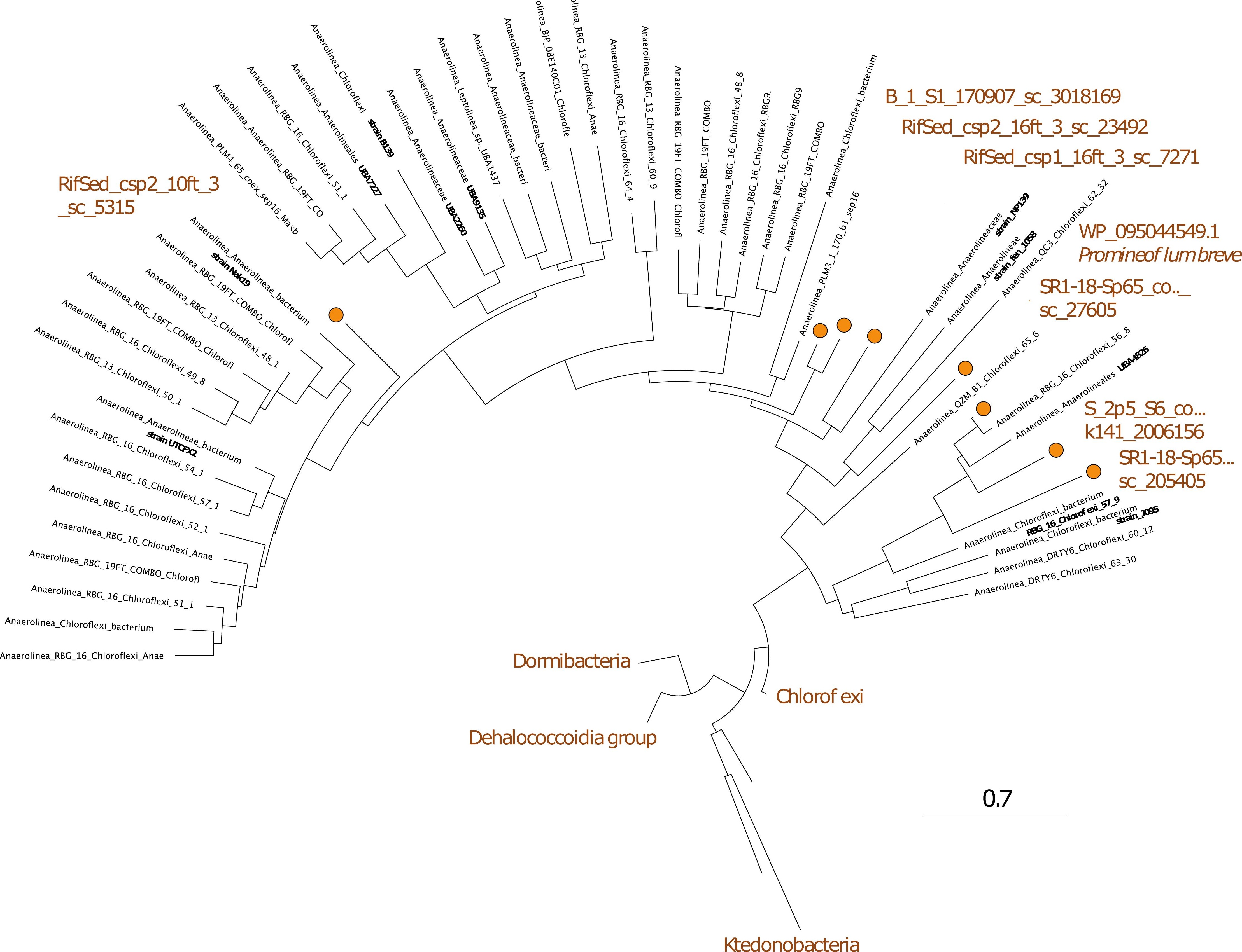




\section{4_0903_08_sc_855}

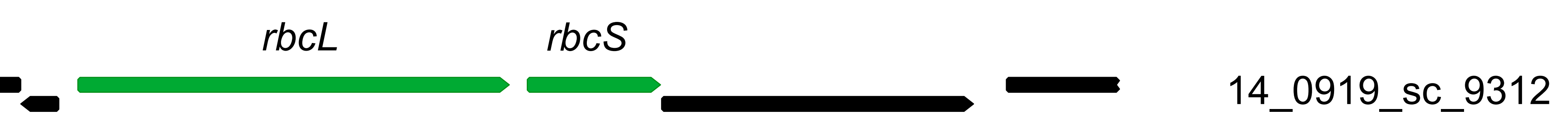

$r b c L \quad r b c S$

2013_60cm_sc_21451

$r b c L \quad r b c S$
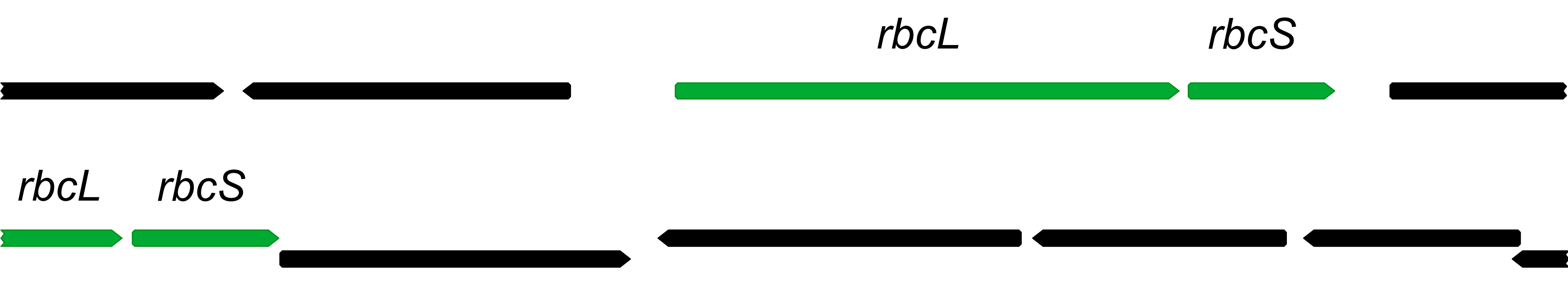

2013_100cm_sc_7750

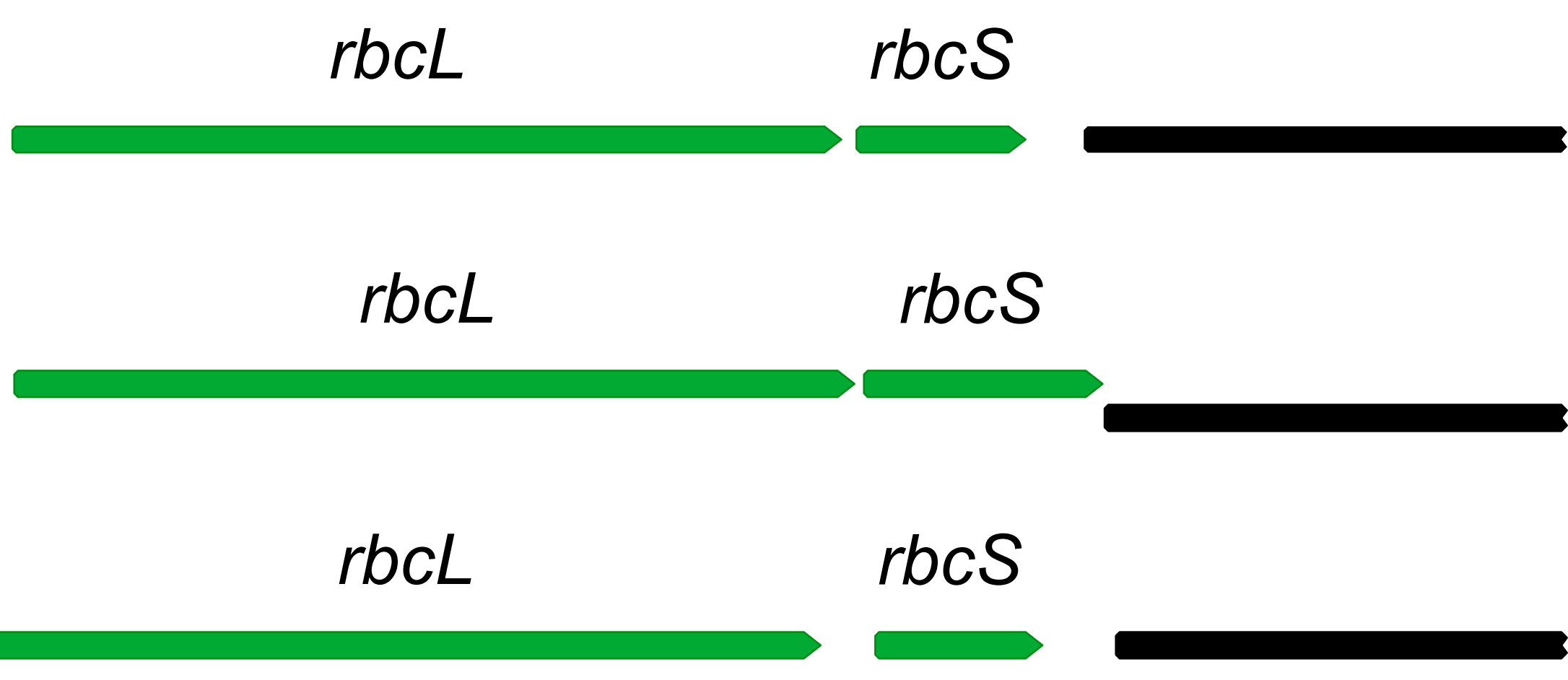

CG_IMS_sc_7750

csp2_sc_11

GD18_4_SC_201747

$r b c L$

$r b c S$

RBG_16_sc_12561

$r b c L \quad r b c S$

RGB_16_sc_19821

$r b c L$

$r b c S$

Sage1_sc_8345 


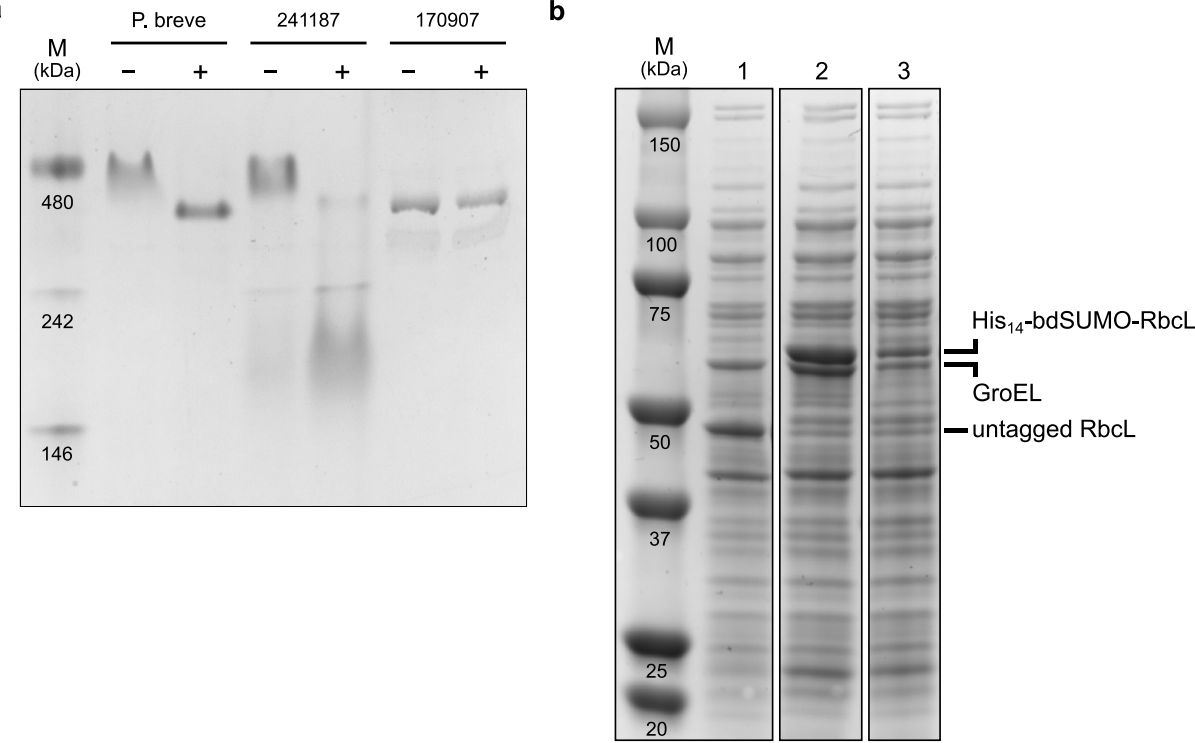


a

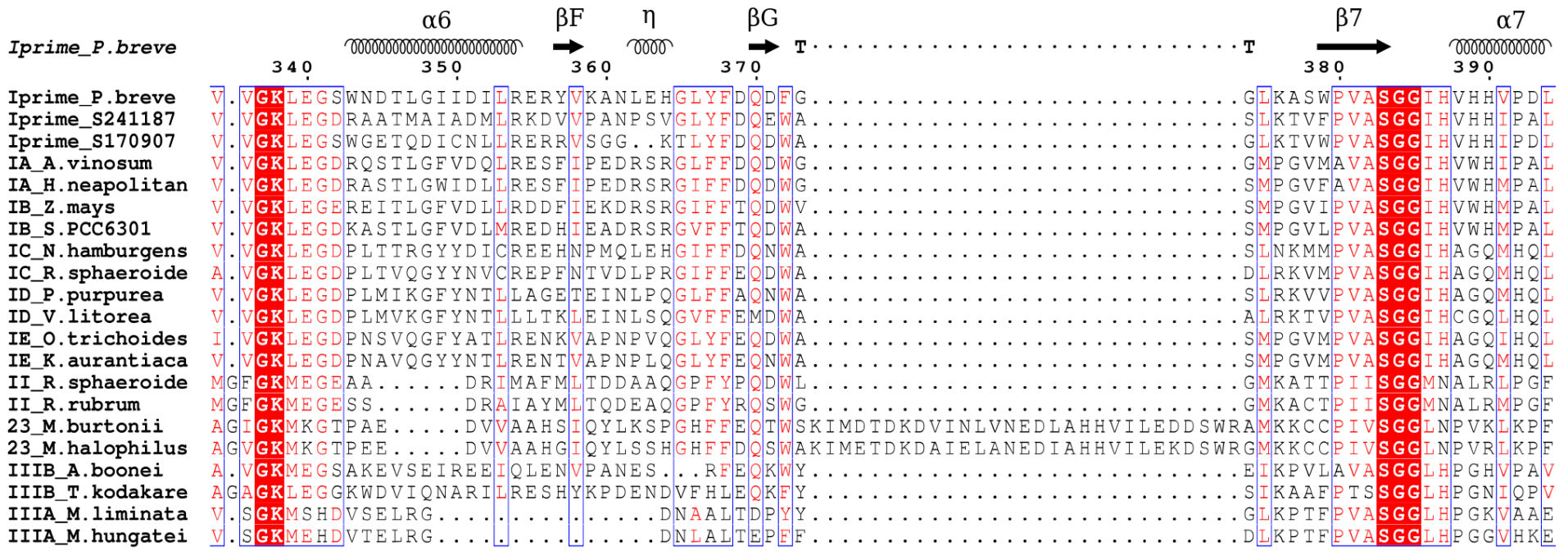

loop 6

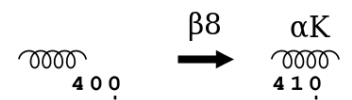

$\alpha 8$ wकw
Rubisco assembly domain (Form II/III)

Iprime_P.breve

Iprime_P.breve Iprime_S241187 Iprime_S170907

IA_A.vinosum IA_H.neapolitan IB_Z.mays IB_S.PCC6301 IC_N.hamburgens IC_R.sphaeroide ID_P.purpurea ID_V.litorea IE O.trichoides IE_K . aurantiaca II_R.sphaeroide II_R.rubrum 23_M.burtonii 23_M.halophilus IIIB_A.boonei IIIB_T.kodakare IIIA_M.liminata IIIA_M.hungatei

b

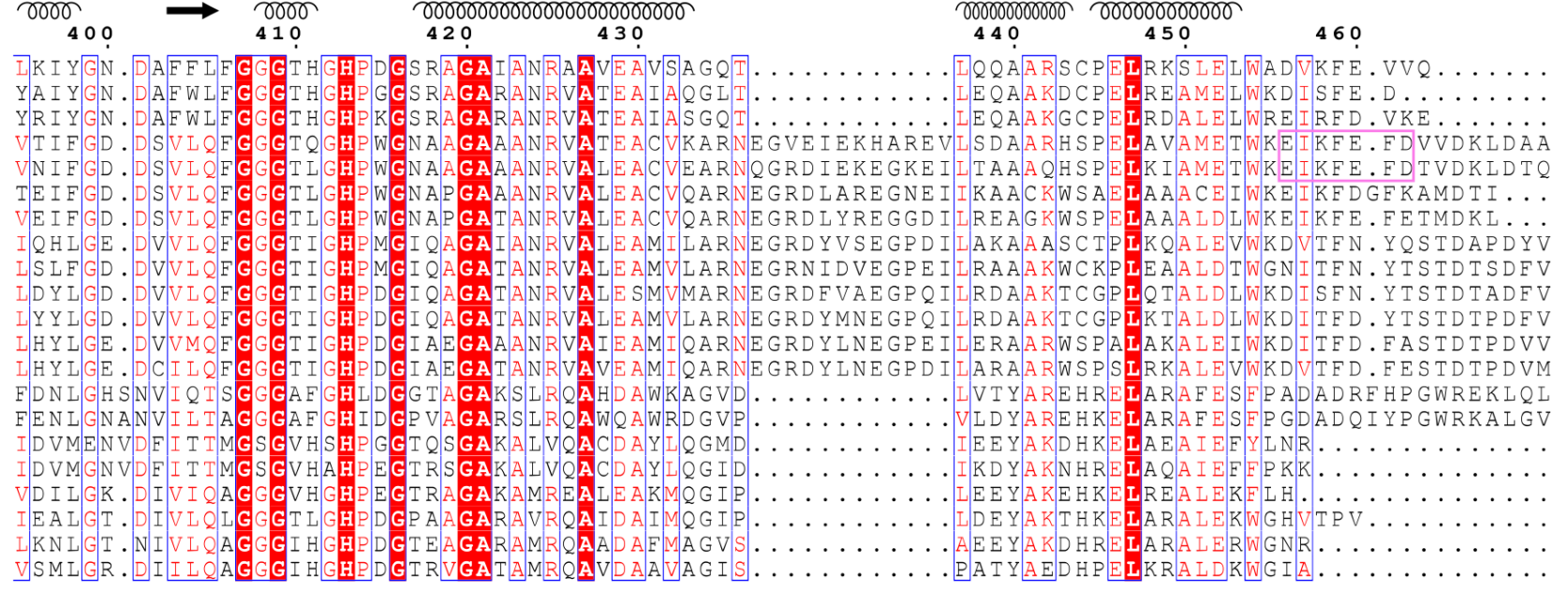

C-terminal ext. (Form I)

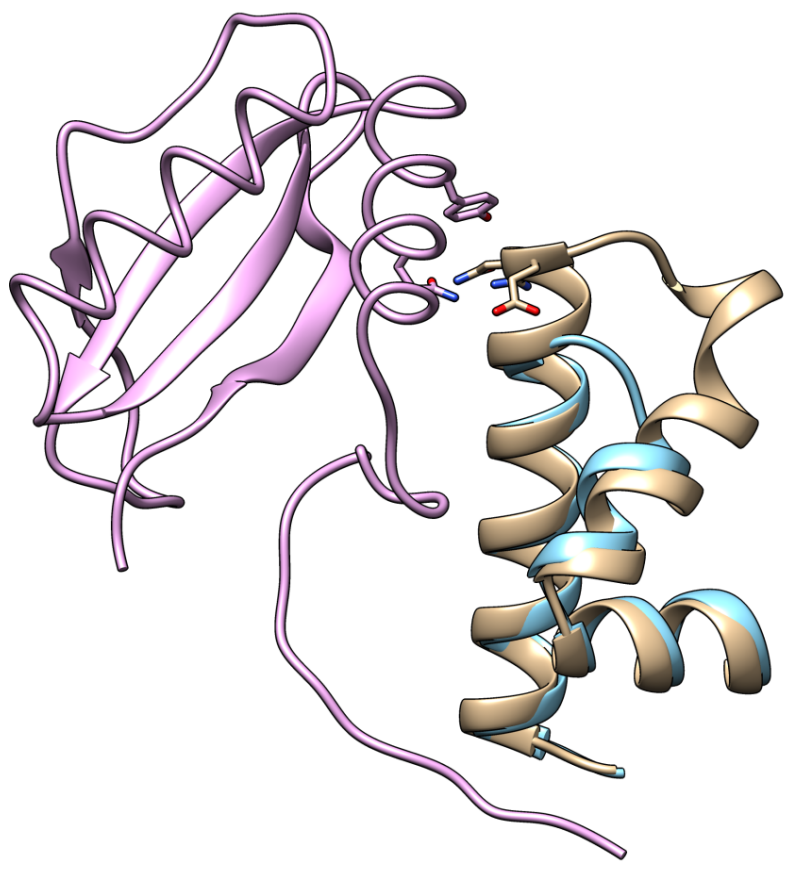



$100 \AA$

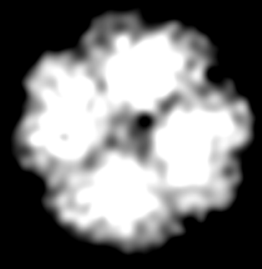

1

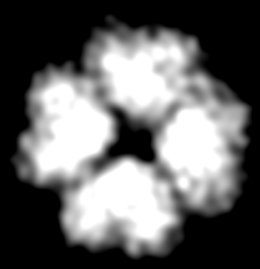

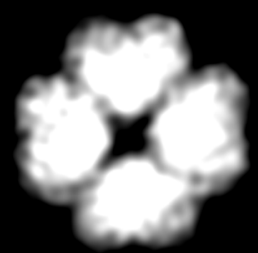

2

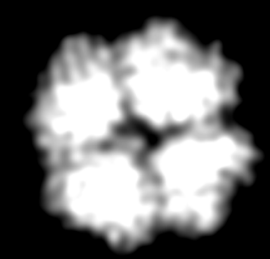

3

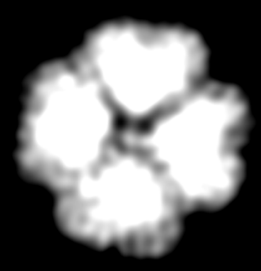

6 
Form I Syn6301 Form I'P.breve

Form I Syn6301 Form I'P.breve
1

1 MAIHNPLAGPKTVKARPTAELSDAYKAGVRAYAVDYYVPDYIPQDTDLLCAFRIQPR-GV

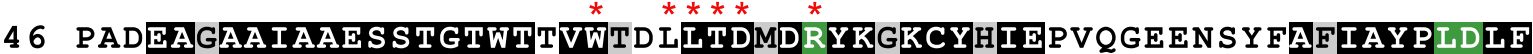
60 DMIEAAAAVAAESSTGTWTEVWSNQLTDIDFYKAKVYAITG- - - - DIAYIAYPLDLF

Form I Syn6301 106 EFGSVTNILTSIVGNVFGFKAIRSLRLEDIRFPVALVKTFOGPPHGIQVERDLLNRYGRP Form I'P.breve 113 EFNSVVNIMSSIVGNVFGFKAVGALRLEDMR I PLALVK TFPGPRVGIYDERVWSNKWDRP

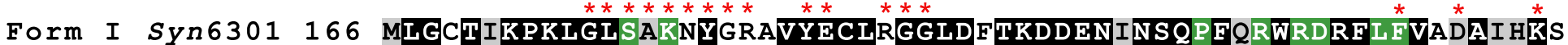
Form I'P.breve 173 IIGGTVKPKLGLSPKAYSTIIYECLSGGLDTSKDDENMNSQPESRWRDRFMYAQEAVDRA

Form I Syn6301 226 QAETGEIKGHYLNVTAPTCERMMKRAEFAKELGMPIIMHDFLTAGFTANT T LARWCRDNG Form I'P.breve 233 AAETNEFKGHWHNVTAGSTEESLRRLEYAELGSRMVMFDFLTAGFAASADIFKRAGELD

Form I Syn6301 286 VLLHIHRAMHAVIDRQRNHGIHFRVLARCLRLSGGDHLHSGTVVGKLEGDKASTLGFVDL Form I'P.breve 293 MIVHCHRAMHAVFTRQANHGIAMRVVAKWLRLTGGDHLHTGTVVGKLEGSWNDTLGIIDI

Form I Syn6301 346 MREDHIEADRSRGVFFTQDKASMPGVLPVASGGIHVWHMPALVEIFGDDSVLQFGGGTLG Form I'P.breve 353 LRERYVKANLEHGLYFDQDFGGLKASWPVASGGIHVHHVPDLLKIYGNDAFFLFGGGTHG

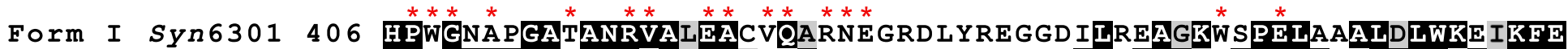
Form I'P.breve 413 HPDGSRAGAIANRAAVEAVSAG---------QTLQQAARSCPELRKSLELWADVKFE

Form I Syn6301 466 FETMDKL Form I'P.breve 461 VVQ----
Syn6301 dimer-dimer interactions

C

b

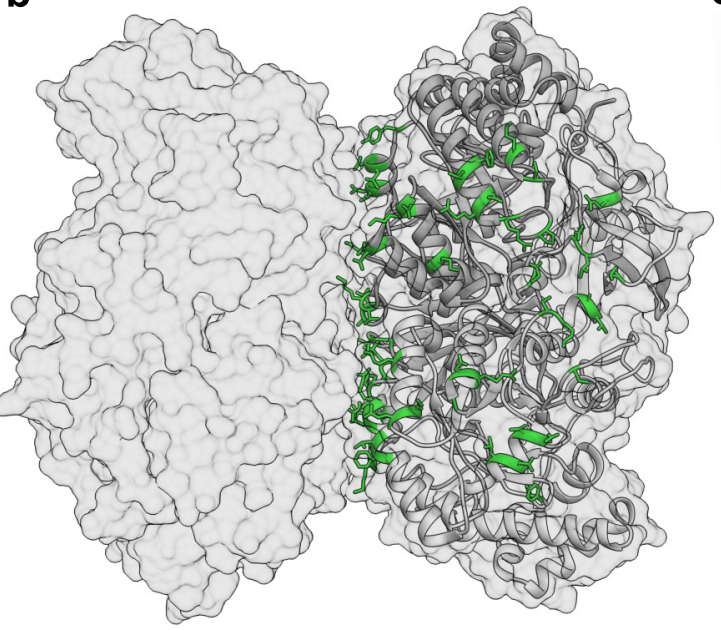

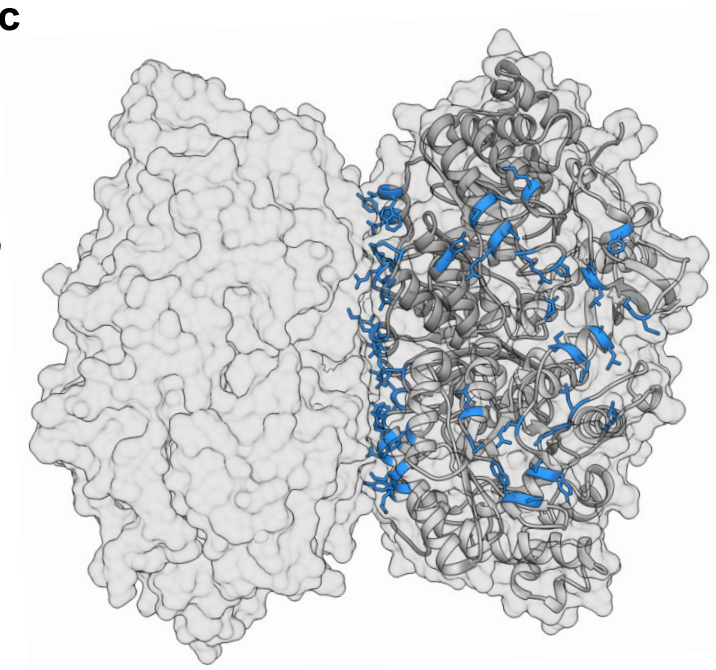

P. breve dimer-dimer interactions

Syn6301 RbcL-RbcS interactions

d

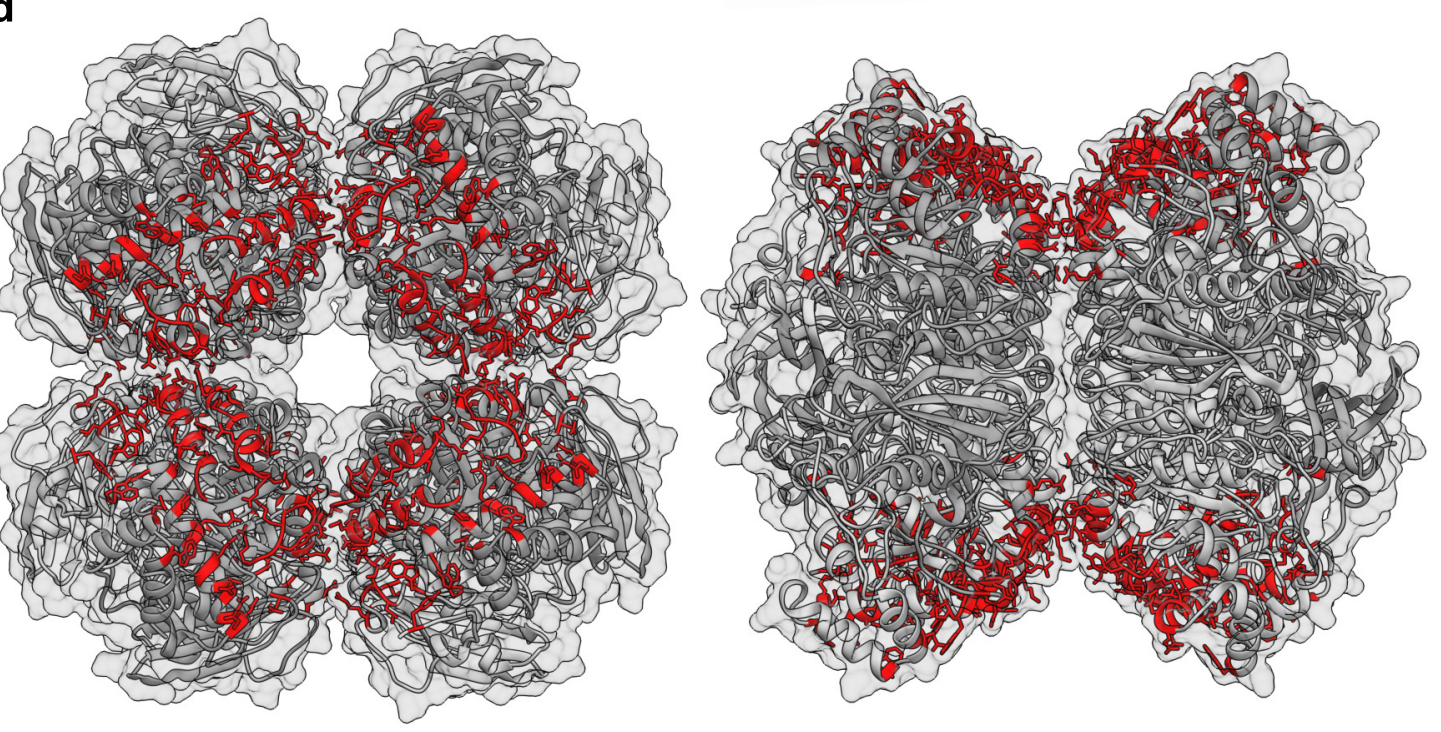




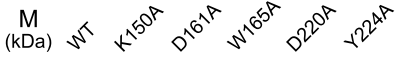

720

480

242

146

66

20 


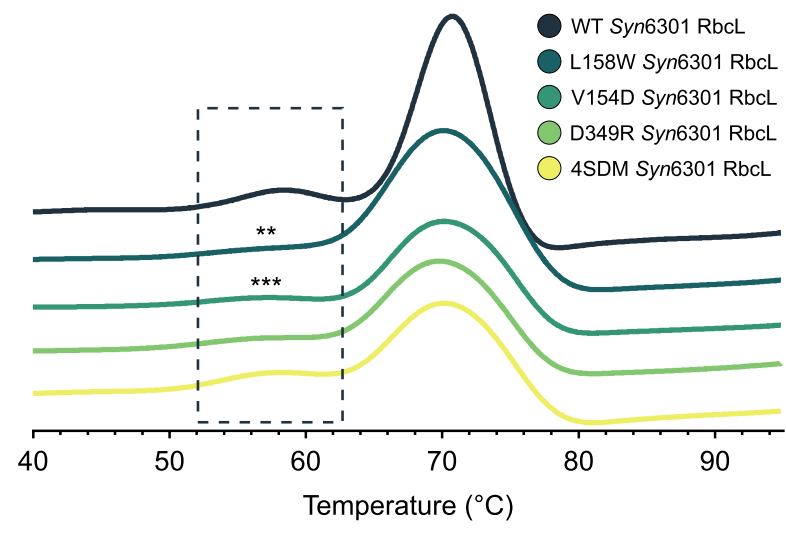

\begin{tabular}{lccc}
\hline Rubsico & $\operatorname{Tm}\left({ }^{\circ} \mathbf{C}\right)$ & $\boldsymbol{n}$ & p-value \\
\hline WT Syn6301 RbcL & $58.6 \pm 0.2$ & 4 & ref. \\
L158W Syn6301 RbcL & $57.7 \pm 0.3$ & 4 & 0.0037 \\
V154D Syn6301 RbcL & $57.3 \pm 0.3$ & 4 & 0.0007 \\
D349R Syn6301 RbcL & $57.9 \pm 0.9$ & 4 & ns \\
4SDM Syn6301 RbcL & $58.6 \pm 0.1$ & 4 & ns \\
\hline
\end{tabular}

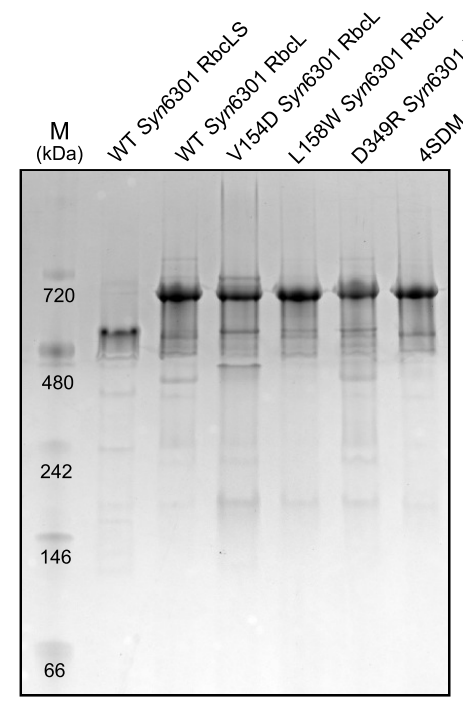

